\title{
Substantivalist and Relationalist Approaches to Spacetime
}

\author{
Oliver Pooley \\ Oriel College, Oxford ${ }^{* \dagger}$
}

6 February 2012

\section{Contents}

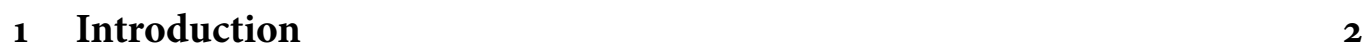

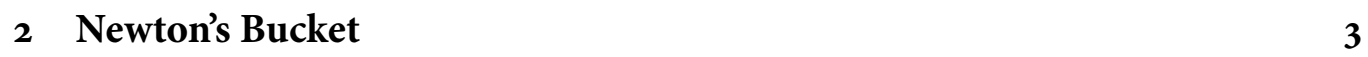

3 The Puzzle of Galilean Invariance 7

$3.1 \quad$ Spacetime and Dynamical Symmetries. . . . . . . . . . . . . . . 7

3.2 The Kinematic Shift Argument $\ldots \ldots \ldots$. . . . . . . . . . 9

4 Spacetime Substantivalism 11

$4.1 \quad$ Neo-Newtonian spacetime. . . . . . . . . . . . . . . . 11

4.2 Symmetries Revisited . . . . . . . . . . . . . . . . . 13

$4.3 \quad$ Relativistic Spacetimes . . . . . . . . . . . . . . . . . 16

5 Reasons to be a Relationalist? 20

$5.1 \quad$ A Failure of Rationality? . . . . . . . . . . . . . . . . . . 20

5.2 The Spacetime Explanation of Inertia . . . . . . . . . . . . 22

* To appear in R. Batterman (ed.), The Oxford Handbook of Philosophy of Physics.

${ }^{\dagger}$ Versions of material on which parts of this chapter are based were presented in Vancouver, Leeds, Minneapolis, Oxford and Florence. I am grateful to members of those audiences for questions and criticism. Particular thanks are due to Harvey Brown, David Wallace, Ulrich Meyer, Julian Barbour, Cian Dorr, Syman Stevens and Julia Bursten for comments on drafts of the paper that led to corrections and improvements. Any errors remaining are, of course, mine. Work for this chapter was partially supported by a Philip Leverhulme Prize and MICINN project FI2008-06418-C03-03, which I gratefully acknowledge. Finally, special thanks are due to the editor for his forbearance. 
6 Three Varieties of Relationalism 26

6.1 Enriched Relationalism . . . . . . . . . . . . . . . . . 27

$6.1 .1 \quad$ Classical Mechanics . . . . . . . . . . . . . . . . . 27

$6.1 .2 \quad$ Relativity . . . . . . . . . . . . . . . . 35

$6.2 \quad$ Barbour's Machian Relationalism . . . . . . . . . . . . . . . 39

6.3 Have-it-all Relationalism . . . . . . . . . . . . . . . . . . . . . 47

6.3.1 The Regularity Approach to Relational Spacetime . . . . . 48

6.3.2 The Dynamical Approach to Relativity . . . . . . . . . . 52

7 Substantivalism in Light of the Hole Argument 58

\section{Introduction}

A significant component of the philosophical interpretation of physics involves investigation of what fundamental kinds of things there are in the world if reality is as physics describes it to be. One candidate entity has proven perennially controversial: spacetime. ${ }^{1}$ The argument about whether spacetime is an entity in its own right goes by the name of the substantivalist-relationalist debate. Substantivalists maintain that a complete catalogue of the fundamental objects in the universe lists, in addition to the elementary constituents of material entities, the basic parts of spacetime. Relationalists maintain that spacetime does not enjoy a basic, non-derivative existence. According to the relationalist, claims apparently about spacetime itself are ultimately to be understood as claims about material entities and the possible patterns of spatiotemporal relations that they can instantiate.

In his Principia, Newton famously distinguished absolute from relative space and states that the former "of its own nature without reference to anything external, always remains homogeneous and immovable" (Newton, 1999, 408). Newton's description of, and arguments for, absolute space are commonly (and rightly) taken to be a statement and defence of substantivalism. In Section 2, I consider Newton's reasons for postulating absolute space before examining, in Section 3 , one of the strongest argument against its existence, the so-called kinematic shift argument. These arguments highlight the close connection between the spatiotemporal symmetries of a dynamical theory and the spacetime ontology that the theory is naturally interpreted as committed to. It turns out that the Galilean covariance of Newtonian mechanics tells against both substantival space and the most obvious relationalist alternative.

\footnotetext{
${ }^{1}$ Strictly speaking, the controversy has concerned two candidate entities. Prior to Minkowski's reformulation of Einstein's special theory of relativity in four-dimensional form, the debate was about the existence of space. Since then, the debate has been about the existence of spacetime. For the sake of brevity, I will often only mention spacetime, leaving the "and/or space" implicit.
} 
With hindsight, the natural substantivalist response to this predicament is to jettison space for spacetime. Section 4 reviews orthodox spacetime substantivalism. The most defensible substantivalist interpretation of Newtonian physics has much in common with a very natural interpretation of relativistic physics. From this perspective, our current best theory of space and time vindicates Newton rather than his relationalist critics, contrary to what early philosophical interpreters claimed (e.g. Reichenbach, 1924).

Section 5 is a sceptical review of two antisubstantivalist themes that motivate some contemporary relationalists. Its conclusion is that the aspiring relationalist's best hope is Ockham's razor, so the focus shifts onto the details of relationalists' proffered alternatives to substantivalism. The move to a four-dimensional perspective expands the range of possibilities available to the classical relationalist. In Section 6 I distinguish three strategies that the relationalist can pursue in the face of the challenge posed by Galilean covariance and consider how the corresponding varieties of relationalism fare when one moves from classical to relativistic physics. It turns out that a number of well-known relationalist views find a natural home in this framework.

A review of the substantivalist-relationalist debate cannot get away without mention of Earman and Norton's (in)famous adaptation of Einstein's Hole Argument. In the final section, I highlight what many see as the most promising substantivalist response and relate it to so-called structural realist approaches to spacetime.

\section{Newton's Bucket}

Newton's best-known discussion of absolute space comes in a scholium to the definitions at the start of the Principia. According to the once-standard reading, Newton's purpose in the Scholium is to argue for the existence of substantival space via the existence of absolute motion, which he supposedly takes to be established by his famous bucket experiment and two-globes thought experiment (see, e.g., Sklar, 1974, 182-4). While there is now widespread agreement that this account badly misrepresents Newton's arguments, there is less consensus over how they in fact should be understood. ${ }^{2}$ Thanks to Koyré (1965) and Stein (1967), it is now recognised that, in order to understand Newton's Scholium, one has to appreciate that Newton was in large part reacting to Descartes' claims about the nature of motion. I briefly review the relevant Cartesian background before giving an account of Newton's arguments that is essentially in agreement with that of Rynasiewicz (1995).

\footnotetext{
${ }^{2}$ For a varied sample of competing interpretations, see Laymon (1978), Rynasiewicz (1995, 2004) and DiSalle 2002 2006), who goes so far as to claim that Newton was not a substantivalist.
} 
Descartes was one of the first natural philosophers to put the principle of inertiathe claim that bodies unaffected by net external forces remain at rest or move uniformly in a straight line-at the centre of his physics (Descartes, 1644, II; 37, 39). At the same time, and apparently without recognising the problem, he espoused an account of motion that is hopelessly incompatible with it. Descartes distinguishes motion in an everyday sense of the term from motion in a strict, philosophical sense. Motion in the ordinary sense is said to be change of place (ibid., II; 24) and Descartes gives a relational definition of a body's place in terms of that body's position relative to external reference bodies regarded as immobile (ibid., II; 10, 13). Which bodies are to be treated as reference bodies is an arbitrary matter. Descartes' ordinary notion of motion is therefore a relative one: a given body may be said to be moving uniformly, non-uniformly or not moving at all, depending on which other bodies are taken to be at rest (ibid., II; 13). In contrast, Descartes' definition of motion in the strict sense, while still relational, was supposed to secure a unique proper motion for each body (ibid., II; 31). True motion is defined as "the transfer of one piece of matter, or of one body, from the vicinity of the other bodies which are in immediate contact with it, and which are regarded as being at rest, to the vicinity of other bodies" (ibid., II; 25, emphasis in the original).$^{3}$

Newton gave a single definition of motion, as change of place, but he also recognised two kinds of motion, depending on whether the places in question were the parts of a relative space (defined in terms of distances relative to material reference bodies) or the parts of substantival space. Newton's relative motion, therefore, corresponds closely to Descartes' motion in the ordinary sense. It is the motion we most directly observe and, Newton agreed, it is what we mean by "motion" in everyday contexts. But, he insisted, when it comes to doing physics, we need to abstract from such observations and consider a body's true motion, which, he argued, has to be defined in terms of an independently-existing absolute space.

Newton's arguments appeal to alleged "properties, causes and effects" of true motion. His aim is to show that various species of relative motion, including Cartesian proper motion (though this is not targeted by name), fail to have the requisite characteristics. If one assumes, as Newton tacitly did, that true motion can only be some kind of privileged relative motion or else is motion with respect to an

\footnotetext{
${ }^{3}$ In 1633, on hearing of the Church's condemnation of Galileo for claiming that the Earth moved, Descartes suppressed an early statement of his physics, which did not contain his later relational claims about the nature of motion. It is frequently (and plausibly) conjectured that Descartes' official views on motion were devised to avoid Church censure. However, the precise manner in which Descartes' definitions secure the Earth's lack of true motion suggest that he was genuinely committed to a relational conception of motion. What does the work in securing the Earth's rest is not that, in Descartes' cosmology, there is no relative motion with respect to immediately contiguous bodies (Descartes explicitly says there is such motion; ibid. III; 28); it is that Cartesian true motion is motion with respect to those contiguous bodies that are regarded as at rest.
} 
independently existing entity, Newton's preferred option wins by default. That each body has a unique, true motion and that such motion has the purported properties, causes and effects, are unargued assumptions.

Newton's claims about the properties of true motion arguably beg the question against the Cartesian. The arguments from causes and effects are more interesting, both because they connect with physics and because their premises were accepted by the Cartesians. The particular effect of true motion that Newton cites is almost an immediate corollary of the principle of inertia: bodies that are undergoing genuine circular motion "endeavour to recede from the axis" because, at each instant, their natural, inertial motion is along the tangent at that point; they only follow their curved path because of the application of a centripetal force.

Descartes fully endorsed these claims about circular motion. Indeed, they formed a central component of his model of planetary motions and the cosmos (e.g. Descartes, 1644, III; 58-62). His definition of true motion, however, fails to fit these phenomena, as Newton's bucket experiment illustrates. Newton asks us to consider a water-filled bucket suspended by a wound cord. Once released, as the cord unwinds, the bucket starts to rotate. Initially, the water is at rest and its surface is flat. As friction gradually transfers the bucket's motion to the water, the water's surface becomes ever more concave until its rate of rotation reaches a maximum and it is comoving with the bucket. The concavity of the water's surface reveals its endeavour to recede from the axis of rotation. According to Descartes' definition of true motion, however, the water is at rest both before the bucket is released and at the end of the experiment, when the water and bucket are once again at relative rest, even though the water now manifests an effect of true rotation. It might also seem that Descartes should count the water as truly moving just after the bucket has been released, because it is transferred from the vicinity of bodies in immediate contact with it (viz., the sides of the bucket), even though, at this stage, the water's surface is flat. The effects of true motion are not correlated with Descartes' definition of true motion in the way they are supposed to be. Newton concludes that the effect revealing true rotation "does not depend on the change of position of the water with respect to [immediately] surrounding bodies, and thus true circular motion cannot be [defined in terms of] such changes of position" (Newton, 1999, 413) ${ }^{4}$

\footnotetext{
${ }^{4}$ The paragraph describing the bucket experiment completes Newton's arguments for his account of true motion in terms of absolute space but it is not the end the Scholium. After a brief paragraph that explicitly concludes: "Hence relative quantities are not the quantities themselves, whose names they bear, but are only sensible measures of them", there follows a long, final paragraph describing a thought experiment involving two globes attached by a cord in a universe in which no other observable objects exist. The purpose of this thought experiment is not to further argue for absolute space by, e.g., describing a situation in which there is absolute motion (revealed by a tension in the cord) and yet no relative motion whatsoever. Instead, Newton's purpose is to demonstrate how true motion can (partially) be empirically determined, despite the imperceptibility of the space with respect to which it is defined: the tension in the cord is a measure of the rate of rotation and, by
} 
The Principia's Scholium on space, time and motion is no longer our only source for Newton's views on these topics. In the 1960s, a pre-Principia manuscript, known after its first line as De Gravitatione, was published for the first time (Newton. 2004). In De Grav, Newton quite explicitly targets Descartes, and one argument is particularly telling.$^{5}$ Newton points out that, according to Descartes' definition of motion, no body has a determinate velocity, and there is no definite trajectory that it follows. From moment to moment a body's motion is defined with respect to those bodies in immediate contact with it, which (for any body in motion) change from moment to moment. There is nothing in this picture that allows us to identify at some time the exact places through which a body has travelled and so a fortiori nothing that can tell us whether these places constitute a straight line which the body has traversed at a uniform rate. Descartes' account of true motion, therefore, cannot secure a fact of the matter about whether a body is moving uniformly, as the principle of inertia requires. Newton concludes: "So it is necessary that the definition of places, and hence of local motion, be referred to some motionless being such as... space in so far as it is seen to be truly distinct from bodies" (Newton. 2004, 20-1).

Talk of a "being" that is "truly distinct from bodies" indicates that Newton's alternative to Cartesian motion involves a variety of substantivalism. The waters are muddied, however, by Newton's explicit denial in De Grav that space is a substance. Newton's position does qualify as version of substantivalism as defined above: according to Newton, space is a genuine entity of a fundamental kind. Newton's denial that space is a substance comes in a passage where he also denies both that it is merely a property ("accident") and that it is "nothing at all". In fact, of the three categories-substance, accident or nothing-Newton states that space is closest in nature to substance. His two reasons for denying that space is a substance relate only to how this category was understood in the then-dominant Scholastic tradition. In particular, space was disqualified from being a substance because, on Newton's view, it does not act and because, in a certain rather technical sense, Newton did not regard it as a self-subsistent entity. ${ }^{6}$

In postulating space as an entity with its own manner of existence, Newton was directly following a number of the early modern atomists, such as Patrizi (1943. 227, 240-1), Gassendi (see, e.g., Grant, 1981, 209) and Charleton (1654, 66). These authors all treated space as more substantial than traditional Aristotelian substances. And there are striking structural parallels between some of the arguments in De

measuring how this tension changes as different forces are applied to opposite faces of the globes, one can also determine the axis and sense of the rotation.

${ }^{5}$ As has been emphasised by Stein $(1967,269-71)$; the argument is also singled out by Barbour (1989. 616-7).

${ }^{0}$ Since Newton held that everything that exists exists somewhere, the existence of any other being entails the existence of space (see Stein, 2002. 300, n. 32, for further discussion). 
Grav and those in Charleton's book, which we know from one of Newton's early notebooks that Newton had studied. The conclusion must be that in postulating substantival space Newton adopted, albeit for truly original reasons, a metaphysical package already very much on the table.

\section{The Puzzle of Galilean Invariance}

For Newton's first two laws of motion to make sense, there needs to be a fact of the matter about whether a body's motion is uniform and, if it is not, a quantitative measure of how it is changing. Newton recognised that Descartes' definitions of motion failed to secure this and signed up to a metaphysics that underwrites the required quantities. There is a sense, though, in which Newton's absolute space underwrites too much. The problem arises because of the symmetries of Newtonian mechanics, in particular its Galilean invariance.

\subsection{Spacetime and Dynamical Symmetries}

The relevant notions of symmetry can be introduced in terms of coordinate transformations. Given our topic, a little care is needed because the substantivalist and the relationalist do not share a conception of a coordinate system. Roughly speaking, a spacetime coordinate system is a map from spacetime into $\mathbb{R}^{4}$ but, of course, only the substantivalist thinks of spacetime as an genuine entity. 7 The relationalist thinks of a coordinate system as assigning quadruples of real numbers $(t, x, y, z)=(t, \vec{x})$ to material events rather than to spacetime points. And, whereas the substantivalist will view every quadruple of a coordinate system as assigned to something, the relationalist will view some sets of coordinate values (those that the substantivalist thinks of as assigned to unoccupied regions) as simply not assigned to anything at all.

Despite this difference, both substantivalists and relationalists will view certain coordinate systems as kinematically privileged in the sense of being optimally adapted to the particular spatiotemporal quantities that they each recognise. In the context of classical mechanics, the natural relationalist alternative to Newton's substantivalism is Leibnizian relationalism. $]^{8}$ According to this view, a possible history of the universe is given by a sequence of relative particle configurations: the primitive spatiotemporal

\footnotetext{
${ }^{7}$ Recall, also, that for the Newtonian substantivalist the basic entity is still space. Until spacetime substantivalism is explicitly introduced in Section 4 . reference to spacetime points should be understood as reference to ordered pairs of point-like substantival places with instants of time, and reference to point-like material events as reference to instantaneous states of persisting point particles.

${ }^{8}$ This terminology is established (see. e.g., Maudlin 1993, 187). No suggestion that the historical Leibniz was a Leibnizian relationalist is intended.
} 
facts about the universe are comprised solely of facts about the instantaneous relative distances between particles (assumed to obey the constraints of Euclidean geometry) and facts about the time intervals between the successive instantaneous material configurations. The ways in which a coordinate system can be adapted to these quantities is straightforward. The time coordinate, $t$, is chosen so that, for any material events $e$ and $e^{\prime}$, the difference, $t(e)-t\left(e^{\prime}\right)$, corresponds to the temporal interval between $e$ and $e^{\prime}$, and is positive or negative according to whether $e$ occurs later or earlier than $e^{\prime}$. Finally, spatial coordinates are chosen so that, for all particles $i, j$ and for all times, $\sqrt{\left(x_{i}-x_{j}\right)^{2}+\left(y_{i}-y_{j}\right)^{2}+\left(z_{i}-z_{j}\right)^{2}}=r_{i j}$, where $r_{i j}$ is the instantaneous inter-particle distance between $i$ and $j$. Assuming that these distances evolve smoothly over time, one also requires that each particle's spatial coordinates are smooth functions of the time coordinate.

Coordinate systems that encode the Leibnizian relationalist quantities in this way are sometimes known as rigid Euclidean coordinate systems (Friedman, 1983. 82). If a particular coordinate system $(t, \vec{x})$ satisfies these constraints then so will any $\left(t^{\prime}, \vec{x}^{\prime}\right)$ related to it by a member of the Leibniz grout $\rrbracket^{9}$ of transformations:

$$
\begin{aligned}
\vec{x}^{\prime} & =\mathbf{R}(t) \vec{x}+\vec{a}(t), \\
t^{\prime} & =t+d .
\end{aligned}
$$

$\mathbf{R}(t)$ is an orthogonal matrix that implements a time-dependent rotation. The components of $\vec{a}(t)$ are smooth functions of time that implement an arbitrary timedependent spatial translation and $d$ is an arbitrary constant that changes the choice of temporal origin.

The manner in which a coordinate system can be adapted to the Newtonian's spatiotemporal quantities is very similar. Mutatis mutandis, the substantivalist imposes the same constraints as the relationalist, although now the spatial and temporal distance relations to which the coordinate values are to be adapted hold between the points of spacetime rather than (only) between material events. ${ }^{10}$ There are also the substantivalists' trademark "same place over time" facts to encode. Here we simply require that the spatial coordinates of each point of space remain constant. The transformations that relate coordinate systems adapted to the full set of Newtonian spatiotemporal quantities form a proper subgroup of the Leibniz group (it might appropriately be labelled the Newton group ${ }^{11}$ ) since the only rotations and

\footnotetext{
${ }^{9}$ There is no established label for these transformations. I follow Bain (2004 350, fn 6); see also Earman (1989 30-1). Ehlers (1973a. 74) calls this group of transformations the kinematical group.

${ }^{10}$ Subject to the qualifications in footnote 7

${ }^{11} \mathrm{Cf}$. Earman (1989 34). Ehlers $(1973 a$. 74) follows Weyl in referring to this group of transformations as the elementary group.
} 
spatial translations that preserve the extra constraint are time-independent:

$$
\begin{aligned}
\vec{x}^{\prime} & =\mathbf{R} \vec{x}+\vec{c}, \\
t^{\prime} & =t+d .
\end{aligned}
$$

Identified in this way, the Leibniz and Newton groups are examples of spacetime symmetry groups: they are groups of transformations that preserve spatiotemporal structure (as encoded in coordinate systems). A conceptually distinct route to identifying special classes of coordinate transformations goes via the dynamical laws of a particular theory. A dynamical symmetry group is a group that preserves the form of the equations that express the dynamical laws. Since the Leibnizian relationalist holds that every rigid Euclidean coordinate system is optimally adapted to all the real spatiotemporal quantities, they might expect such coordinate systems to be dynamically equivalent. In other words, it is natural for someone who thinks that the Leibniz group is a spacetime symmetry group to expect it to be a dynamical symmetry group as well. This might indeed be the case if, for example, the dynamical laws dealt directly with the relative distances between bodies. But Newton's laws do not. Instead they presuppose that individual bodies have determinate motions independently of their relations to other bodies. If Newton's laws take their canonical form in a given coordinate system $K$ (which, we may imagine, is adapted to Newtonian space and time), then they will not take the same form in a coordinate system, $K^{\prime}$, related to $K$ by an arbitrary member of the Leibniz group. The equations that hold relative to $K^{\prime}$ will involve additional terms corresponding to source-free 'pseudo forces' that, the Newtonian maintains, are artefacts of $K^{\prime}$ 's acceleration with respect to substantival space.

\subsection{The Kinematic Shift Argument}

The mismatch between dynamical symmetries and (what Leibnizian relationalists regard as) spacetime symmetries is a problem for the relationalist. But a similar problem afflicts the Newtonian substantivalist. Whilst the Newton group is a dynamical symmetry group of classical mechanics, it is not the full symmetry group. The equations that express Newton's three laws of motion and particular Newtonian force laws (such as the law of universal gravitation) are invariant under a wider range of coordinate transformations, namely those constituting the Galilei group:

$$
\begin{aligned}
\vec{x}^{\prime} & =\mathbf{R} \vec{x}+\vec{v} t+\vec{c}, \\
t^{\prime} & =t+d .
\end{aligned}
$$

As in $(\mathrm{New})$, the rotation matrix is time-independent, but now uniform timedependent translations of the spatial coordinates ("boosts") are allowed. 
Let's stipulate that two coordinate systems are adapted to the same frame of reference if and only if they are related by an element of the Newton group ${ }^{12}$ Two coordinate systems related by a non-trivial Galilean boost are then adapted to different frames of reference. However, if Newton's laws hold with respect to either frame, they hold with respect to both of them. In particular, both frames might be inertial frames in that, with respect to them, force-free bodies move uniformly in straight lines.

This gives rise to the following epistemological embarrassment for the Newtonian substantivalist. Imagine a possible world $W^{\prime}$ just like the actual world except that, at every moment, the absolute velocity of each material object in $W^{\prime}$ differs from its actual value by a fixed amount (say, by two metres per second in a direction due North). $W^{\prime}$ is an example of a world that is kinematically shifted relative to the actual universe. Two kinematically-shifted worlds are observationally indistinguishable because, by construction, the histories of relative distances between material objects in each world are exactly the same. The worlds differ only over how the material universe as a whole is moving with respect to space. Since substantival space is not directly detectible, this is not an observable difference. Further, the Galilean invariance of Newtonian mechanics means that any two kinematically-shifted worlds either both satisfy Newton's laws, or neither does.

The upshot is that the Newtonian substantivalist is committed to the physical reality of certain quantities, absolute velocities, that are in principle undetectable given the symmetries of the dynamical laws. Given how $W^{\prime}$ was specified in the previous paragraph, we know it is not the actual world. But consider a world, $W^{\prime \prime}$, just like the actual world in terms of the relative distances between bodies at each moment but in which, at 12 a.m. on 1st January 2000 , the absolute velocity of the Eiffel Tower is exactly $527 \mathrm{~ms}^{-1}$ due North. For all we know, $W^{\prime \prime}$ is the actual world. ${ }^{13}$ To paraphrase Maudlin (1993, 192), there may be no a priori reason why all physically real properties should be experimentally discoverable but one should at least be uneasy about empirically inaccessible physical facts; ceteris paribus, one should prefer a theory that does without them. ${ }^{14}$

\footnotetext{
${ }^{12}$ In spacetime terms, the notion of a frame of reference implicit in this stipulation corresponds to the following: a fibration of spacetime that specifies a standard of rest; a foliation of spacetime that specifies a standard of distant simultaneity; a temporal metric on the quotient of spacetime by the foliation; and a spatial metric on the quotient of spacetime by the fibration.

${ }^{13}$ Supposing, for the sake of argument, that the actual universe is Newtonian.

${ }^{14}$ In his correspondence with Clarke (Alexander, 1956), Leibniz is sometimes read as offering kinematic-shift arguments somewhat different to the one just sketched. The idea is that kinematicallyshifted possible worlds would violate the Principle of Sufficient Reason (PSR) and the Principle of the Identity of Indiscernibles (PII). Since these principles are $a$ priori true, according to Leibniz, there can be no such plurality of possibilities. A 'Leibnizian' argument from the PSR would ask us to consider what reasons God could have had for creating the actual universe rather than one of its kinematicallyshifted cousins. An argument from the PII would claim that, since kinematically-shifted worlds are
} 
The conclusion of this section is that the Galilean invariance of Newtonian physics poses something of an interpretative dilemma. On the one hand, to make sense of the successful dynamical laws it seems that we have to acknowledge more spacetime structure than the Leibnizian relationalist is prepared to countenance. On the other hand, Newton's manner of securing a sufficiently rich structure introduces more than is strictly required and therefore underwrites empirically undetectable yet allegedly genuine quantities. Absent an alternative way to make sense of Newtonian physics, one might learn to live with Newton's metaphysics. However, the irritant of absolute velocities motivates a search for an alternative. In the next section, I consider a substantivalist way out of the dilemma. Relationalist strategies are explored in Section 6 .

\section{Spacetime Substantivalism}

\subsection{Neo-Newtonian spacetime}

The substantivalist can do away with unwanted absolute velocities by adopting the essentially four-dimensional perspective afforded by spacetime. In this framework, there is an elegant way of characterising a spatiotemporal structure that might seem to be neither too weak nor too strong for Newtonian physics. ${ }^{15}$ The dynamical quantities of classical mechanics presuppose the simultaneity structure, instantaneous Euclidean geometry and temporal metric common to the Leibnizian relationalist and the Newtonian substantivalist. They additionally require some extra transtemporal structure. Geometrically, what's needed is a standard of straightness for paths in spacetime (provided in differential geometry by an affine connection). The possible trajectories of ideal force-free bodies correspond to those straight lines in spacetime that do not lie within surfaces of simultaneity. These straight lines fall into families of non-intersecting lines that fill spacetime. Each family of lines are the trajectories

observationally indistinguishable, they directly violate the PII.

Neither argument is convincing (nor is either faithful to Leibniz; see Pooley (unpublished)). The sense of indiscernbility relevant to kinematic shifts is not that which has been the focus of contemporary discussion of the PII. This takes two entities to be indiscernible just if they share all their (qualitative) properties. In general, two kinematically-shifted worlds do differ qualitatively; given how the qualitative/nonqualitiative distinction is standardly understood, a body's absolute speed is a qualitative property, and differences in absolute velocity are (typically) qualitative differences. Such qualitative differences are empirically inaccessible but, theoretically, they could ground a reason for an all-seeing God's preference for one possibility over another. A PSR dilemma for God is created if we consider kinematically-shifted worlds that differ, not in terms of the magnitude of their objects' absolute velocities, but only over their directions. These are worlds that are qualitatively indistinguishable. Discussion of how the substantivalist should treat these is postponed until Section 7

${ }^{15}$ In fact, it is too strong; see Section 6.1 below. 
of the points of the 'space' of some inertial frame. The resulting spacetime is known as Galilean or neo-Newtonian spacetime (see, e.g., Sklar, 1974, 202-6; Earman, 1989 33).

Now, it is one thing to give a non-redundant characterisation of the spacetime structure that Newtonian mechanics assumes. It is another to provide a satisfactory account of its metaphysical foundations (i.e., of what, in reality, underwrites this structure). The obvious option is to take the unitary notion of spacetime (rather than space and time separately) ontologically seriously. One regards spacetime as something that exists in its own right and which literally has the geometric structure that the affine connection, amongst other things, encodes. In terms of such ontology, one can provide a metaphysical account of the distinction between absolute and relative motion in a way that respects the physical equivalence of inertial frames.

It will facilitate comparison with relativistic theories to introduce a formulation of Newton mechanics that makes explicit reference to this geometrical structure. In abstract terms, physical theories often have the following general form. A space $\mathcal{K}$ of kinematically possible models (KPMs) is first specified. The job of the theory's equations (which relate the quantities in terms of which the KPMs are characterised) is then to single out the subspace $\mathcal{S}$ of $\mathcal{K}$ containing the dynamically possible models (DPMs) ${ }^{16}$ The KPMs can be thought of as representing the range of metaphysical possibilities consistent with the theory's basic ontological assumptions. The DPMs represent a narrower set of physical possibilities.

In a coordinate-dependent formulation of Newtonian theory like that so far considered, the KPMs might be sets of inextendible smooth curves in $\mathbb{R}^{4}$ which are nowhere tangent to surfaces of constant $t$ (where $(t, \vec{x}) \in \mathbb{R}^{4}$ ). The models assign to the curves various parameters $(m, \ldots)$. Under the intended interpretation, the curves represent possible trajectories of material particles, described with respect to a canonical coordinate system, and the parameters represent various dynamically relevant particle properties, such as mass. The space of DPMs consists of those sets of curves that satisfy the standard form of Newton's equations.

In the local spacetime formulation of the theory, one takes the KPMs to be $n$-tuples of the form $\left\langle M, t_{a b}, h^{a b}, \nabla_{a}, \Phi_{1}, \Phi_{2}, \ldots\right\rangle{ }^{17} M$ is a four-dimensional differentiable manifold and $t_{a b}, h^{a b}$ and $\nabla_{a}$ are geometric-object fields on $M$ that encode, respectively, the temporal structure (both the simultaneity surfaces and the temporal

\footnotetext{
${ }^{16}$ These notions are standard, although terminology varies; see, e.g., Anderson $(1967,74)$ and Friedman (1983, 48).

${ }^{1 /}$ A locus classicus in the philosophical literature for a discussion of Newtonian theory formulated in this style is Friedman (1983. Ch. III). I adopt the following widespread notational conventions: Roman indices from the start of the alphabet do not denote components-they are "abstract indices" merely indicating the type of geometric-object field; Greek indices denote that the components of the objects relative to some spacetime coordinate system are being considered; repeated indices indicate a sum over those indices (the Einstein summation convention).
} 
metric), the Euclidean geometry of instantaneous space and the inertial structure (i.e., which paths in spacetime are straight). Together they represent neo-Newtonian spacetime. The "matter fields" $\Phi_{i}$, which represent the material content of the model, can be curves (maps from the real line into $M$, representing particle trajectories) or fields (maps from $M$ into some space of possible values, either encoding force potentials or continuous matter distributions).

The theory's DPMs are picked out by a set of equations that relate these various objects. Some of these will involve both the spacetime structure and the matter fields. For example, Newton's second law becomes:

$$
F^{a}=m \xi^{n} \nabla_{n} \xi^{a}
$$

For those not familiar with the tensor notation, the essential points to note about this equation are the following. $F^{a}$ and $\xi^{a}$ are spacetime four-vectors. $F^{a}$ stands for the four-force on the particle. As in more traditional formulations of Newtonian theories, it will be specified by one or more additional equations. $\xi^{a}$ is the fourvelocity of the particle; it is the tangent vector to the particle's spacetime trajectory if that trajectory has been parameterised by absolute time ${ }^{18}$ Note, in particular, the explicit appearance of $\nabla_{a}$ in the equation. $\xi^{n} \nabla_{n} \xi^{a}$ is the four-acceleration of the particle; it characterises how the particle's trajectory deviates from the adjacent tangential straight line in spacetime (i.e., from the relevant inertial trajectory).

\subsection{Symmetries Revisited}

In Section 3 the Galilei group and the Leibniz group were introduced as sets of coordinate transformations, and the dynamical symmetries of Newtonian mechanics were characterised in terms of the form invariance of its equations. We now see that the implementation of this approach is not completely straightforward: various formulations of Newtonian mechanics involve different sets of equations, and these can have different invariance properties. In particular, when Newton's laws are recast so as to make explicit reference to the geometrical structure of neo-Newtonian spacetime, the resulting equations are either generally covariant (they hold with respect to a set coordinate systems related by smooth but otherwise arbitrary coordinate transformations) or they are coordinate-independent (they directly equate certain geometrical objects, rather than the values of the objects' components in some coordinate system).

There is an alternative way to characterise the symmetries of a spacetime theory. Rather than focusing on the theory's equations, one considers their solutions. Suppose, now, that a group $G$ of maps from spacetime to itself has a natural action on

\footnotetext{
${ }^{18}$ I.e., the trajectory is parametrized so that $t_{a} \xi^{a}=1$, where $t_{a}$ is a one-form related to the temporal metric via $t_{a b}=t_{a} t_{b}$.
} 
the space of KPMs. $G$ is a symmetry of the theory if and only if it fixes the solution space $S \cdot{ }^{19}$ Note the need to relativise this characterisation of symmetry to groups of maps from spacetime to itself. If we were allowed to consider any action of any group on the space of KPMs, the requirement that $S$ be fixed would be too easily satisfied. In particular, for any two points $s_{1}, s_{2}$ in $\mathcal{S}$, we could find a group action on the space of KPMs such that $S$ is fixed and $s_{1}$ is mapped to $s_{2}$. I.e., every point in $\mathcal{S}$ would be mapped to every other by some symmetry transformation or other ${ }^{20}$

If the KPMs and DPMs of Newtonian mechanics are defined according to the first formulation above (as certain classes of curves in $\mathbb{R}^{4}$ ), the symmetry group of the theory turns out, as one might have expected, to be the Galilei group. This is not so if the local spacetime formulation of the theory is adopted. The space of KPMs then carries an action of the diffeomorphism group $\operatorname{Diff}(M)$ : for any $\left\langle M, t_{a b}, h^{a b}, \nabla_{a}, \Phi_{i}\right\rangle \in \mathcal{K}$ and for any $d \in \operatorname{Diff}(M),\left\langle M, d^{*} t_{a b}, d^{*} h^{a b}, d^{*} \nabla_{a}, d^{*} \Phi_{i}\right\rangle \in$ $K^{21}$ It follows from the tensorial nature of the equations that pick out the solution subspace that, if $\left\langle M, t_{a b}, h^{a b}, \nabla_{a}, \Phi_{i}\right\rangle$ satisfies the equations then so does $\left\langle M, d^{*} t_{a b}, d^{*} h^{a b}, d^{*} \nabla_{a}, d^{\star} \Phi_{i}\right\rangle$ (Earman and Norton, 1987, 520). In other words, the full group $\operatorname{Diff}(M)$ fixes $\mathcal{S}$ and therefore counts as a symmetry group of this formulation of the theory.

At this point it might look as if the formulation-dependence that afflicts a definition of dynamical symmetries in terms of the invariance of equations has simply been reproduced at the level of models. We can, however, reintroduce the distinction between spacetime and dynamical symmetries in model-theoretic terms. Our characterisation of the models of a local spacetime formulation of Newtonian mechanics involved distinguishing those geometric-object fields on $M$ that represent spacetime structure from those that represent the material content of spacetime. Let's write $\left\langle M, A_{1}, \ldots, A_{n}, P_{1}, \ldots, P_{m}\right\rangle$ for a generic spacetime model, where the $A_{i}$ s stand for the fields that represent spacetime structure and the $P_{j}$ s stand for the fields that represent the matter content. Recall that the coordinate-dependent definition of spacetime symmetries given in Section 3 required that the encoding

\footnotetext{
${ }^{19}$ In general, an action of group $G$ on a space $\mathcal{K}$ is a function $\phi:(g, k) \in G \times \mathcal{K} \mapsto g \cdot k \in \mathcal{K}$ such that, for all $g, h \in G$ and $k \in \mathcal{K}, g \cdot(h \cdot k)=(g h) \cdot k$ and $e \cdot k=k$, where $e$ is the identity element of $G$. To avoid triviality, we should also require that the action is faithful, i.e., that for any $g$, if $g \cdot k=k$ for all $k, g=e$. $G$ is a symmetry group if and only if $g \cdot s \in \mathcal{S}$ for all $g \in G$ and $s \in \mathcal{S}$. The symmetry group of a theory characterised in this way is referred to as the theory's covariance group by Anderson (1967. 75).

${ }^{20}$ See Gordon Belot's chapter in this volume for further discussion.

${ }^{21}$ The diffeomorphism group Diff $(M)$ is the group of all differentiable one-one mappings from $M$ onto itself. The definition of the map $d^{*}$, which acts on geometrical objects on $M$ and is induced by the manifold mapping $d: p \in M \mapsto d p \in M$, will depend on the type of field. For a scalar field, $\phi$, $d^{*} \phi(d p):=\phi(p)$. The action of $d^{*}$ on scalar fields can then be used to define its action on tensor fields. For example, for the vector field $V$, we require that $\left.d^{*} V\left(d^{*} \phi\right)\right|_{d p}=\left.V(\phi)\right|_{p}$ for all points $p$ and scalar fields $\phi$.
} 
of spacetime structure by the coordinate system was preserved. Analogously, we can identify a theory's spacetime symmetry group as the set of elements of $\operatorname{Diff}(M)$ that are automorphisms of the spacetime structure; i.e., we require that $d^{*} A_{i}=A_{i}$ for each field $A_{i}{ }^{22}$ In the case of our Newtonian theory, the proper subgroup of $\operatorname{Diff}(M)$ that leaves each of $t_{a b}, h^{a b}$ and $\nabla_{a}$ invariant is the Galilei group.

An alternative method of singling out a subgroup of $\operatorname{Diff}(M)$ focuses on the matter fields rather than the spacetime structure fields. Typically the matter content of a solution will have no non-trivial automorphisms and, if it does, there will be other solutions whose matter content does not share these symmetries. We can, however, ask whether a diffeomorphism acting solely on the matter fields maps solutions to solutions. In other words, we pick out a subgroup of $\operatorname{Diff}(M)$ via the requirement that (for a given choice of $A_{i}$ ) for all $\left\langle M, A_{i}, P_{j}\right\rangle \in \mathcal{S},\left\langle M, A_{i}, d^{*} P_{j}\right\rangle$ should also be in $S \cdot{ }^{23}$ Note that, in this definition, $d$ acts only on the matter fields and not on the spacetime structure fields. The subgroup of diffeomorphisms with this property is sometimes identified as a theory's dynamical symmetry group (Earman. 1989. 45-6). Again, in the spacetime formulation of Newtonian theory set in neoNewtonian spacetime, if this group turns out to be the Galilei group one has a perfect match between the spacetime symmetry group and the dynamical symmetry group ${ }^{24}$

The model-theoretic perspective supports the idea that the problems faced by both the Leibnizian relationalist and the Newtonian substantivalist involve mismatches between these two symmetry groups. Whenever the spacetime symmetry group is a proper subset of the dynamical symmetry group, the theory will admit non-isomorphic models whose material submodels are nevertheless isomorphic. (Such models will be related by dynamical symmetries that are not also spacetime symmetries.) This will give rise to supposedly meaningful yet physically undiscoverable quantities: models will differ over some quantities in virtue of different relations of matter to spacetime structure and yet (assuming the material content of the models encompasses all that is observable) such differences will be undetectable. This is exactly the situation of the Newtonian substantivalist, who postulates a richer

\footnotetext{
${ }^{22}$ More carefully, the requirement that $d^{*} A_{i}=A_{i}$ for each $A_{i}$ picks out a specific subgroup of $\operatorname{Diff}(M)$ relative to a particular choice of $A_{i}$. Suppose that $\left\langle M, A_{i}, P_{j}\right\rangle$ and $\left\langle M, A_{i}^{\prime}, P_{j}^{\prime}\right\rangle$ are both models of our theory and that there is a diffeomorphism $\phi: M \rightarrow M$ such that $A_{i}^{\prime}=\phi^{*} A_{i}$ for each $A_{i}, A_{i}^{\prime}$ (all models of a theory set in Galilean spacetime will have this property). Although it will not be true, in general, that $\left\{d \in \operatorname{Diff}(M): d^{*} A_{i}=A_{i}\right\}=\left\{d^{\prime} \in \operatorname{Diff}(M): d^{\prime *} A_{i}^{\prime}=A_{i}^{\prime}\right\}$, the groups will be isomorphic to the same (abstract) group; $c f$. Earman $(1989,45)$.

${ }^{23}$ As noted in footnote 22 different choices of $A_{i}$ will, strictly speaking, yield distinct subgroups of Diff $(M)$ but (for well-behaved theories) these will simply correspond to different representations of the same abstract group.

${ }^{24}$ The dynamical symmetry group of Newtonian theory set in neo-Newtonian spacetime in fact turns out to be a larger group if the theory incorporates gravitation in a field-theoretic way. See pp 31 ff below.
} 
spacetime structure than that of neo-Newtonian spacetime. Models related by Galilean boosts of their material content differ over undetectable absolute velocities.

The mismatch faced by the Leibnizian relationalist is of the opposite kind: the Galilei group is a proper subset of the Leibniz group. Strictly speaking, this is not a case where the dynamical symmetry group of some theory is smaller than the spacetime symmetry group. For that we would need a spacetime formulation of a theory that (i) was set in so-called Leibnizian spacetime and (ii) had the Galilei group as its dynamical symmetry group (e.g., in virtue of an isomorphism between the set of its matter submodels and those of standard Newtonian theory). But the way in which the equations of the spacetime formulation of standard Newtonian theory single out its matter submodels uses the full structure of neo-Newtonian spacetime. The mismatch between the two groups is precisely what stands in the way of constructing such a relational theory.

Before considering the spacetime substantivalist interpretation of relativistic physics, a brief comment on the relation between the two formulations of Newtonian mechanics that I have been discussing: I first characterised the privileged coordinate systems of the coordinate-dependent form of Newtonian physics as those adapted to the spatiotemporal quantities recognised by the Newtonian. From this perspective, the spacetime formulation of the theory simply makes explicit structure that, while implicit, is no less present in the coordinate-dependent, Galilean-covariant formulation of the theory. Suppose one starts with (the coordinate expressions of) the equations of the spacetime formulation of a Newtonian theory. These equations will be generally covariant. However, one can use the symmetries of the theory's spacetime structure to pick out a special class of coordinate systems in which the values of the components of the fields representing the spacetime structure take on constant or vanishing values. In these coordinate systems the otherwise generally covariant equations apparently simplify. In other words, the Galilean covariant equations just are the generally covariant equations, written with respect to coordinate systems that 'hide' the objects that represent spacetime structure. ${ }^{25}$

\subsection{Relativistic Spacetimes}

In the previous section, I maintained that the geometrical structure of neo-Newtonian spacetime featured, implicitly or otherwise, in the various formulations of classical mechanics. A similar claim holds true for special relativity. This is most obvious in

\footnotetext{
${ }^{25}$ This might seem like a banal observation but I take it to be significant because it conflicts with prevalent claims about the meaning of preferred coordinates in non-generally-covariant theories made by, e.g., Rovelli (2004, 87-8) and Westman and Sonego 2009, 1952-3). Their conception of the significance of such coordinates implies that there is a difference in kind between the observables of non-covariant and generally covariant theories. On the view outlined above, there is no such difference.
} 
generally covariant or coordinate-free formulations of the equations of any specially relativistic theory, where the Minkowski metric structure, encoded by the tensor field $\eta_{a b}$, figures explicitly. But it is equally true of the 'standard' formulations of the equations that hold true only relative to privileged inertial coordinate systems related by Lorentz transformations. One can think of these coordinate systems as the spacetime analogues of Cartesian coordinates on Euclidean space: the coordinate intervals encode the spacetime distances via the condition $\left(t_{p}-t_{q}\right)^{2}-\left|\vec{x}_{p}-\vec{x}_{q}\right|^{2}=\Delta s^{2}$. Minkowski geometry is thus implicit in the standard formulation of the laws.

Because of the manner in which spacetime geometry features in the formulation of the laws, substantivalists hold that it explains certain features of the phenomena covered by those laws ${ }^{26}$ Consider, for example, the "twin paradox" scenario. Of two initially synchronized clocks, one remains on Earth while the other performs a round trip at near the speed of light. On its return the travelling clock has ticked away less time than the stay-at-home clock. The geometrical facts behind this phenomenon are straightforward: the inertial trajectory of the stay-at-home clock is simply a longer timelike path than the trajectory of the travelling clock. Note that the substantivalist does not simply assert that the number of a clock's ticks is proportional to the spacetime distance along its trajectory. Clocks are complicated systems the parts of which obey various (relativistic) laws. One should look to these laws for a complete understanding of why the 'ticks' of such a system will indeed correspond to equal temporal intervals of the system's trajectory. But since, for the substantivalist, these laws make (implicit or explicit) reference to independently real geometric structure, an explanation that appeals to the laws will, in part, be an explanation in terms of such geometric structure, postulated as a fundamental feature of reality.

Soon after formulating his special theory of relativity, Einstein began a decadelong quest for a theory of gravitation that was compatible with the new notions of space and time. The triumphant culmination of this effort was his publication of the field equations of his general theory of relativity (GR) in $1915 .{ }^{27}$ These equations, known as the Einstein Field Equations (EFEs), relate certain aspects of the curvature of spacetime, as encoded in the Einstein tensor, $G_{a b}$, to the matter content of

\footnotetext{
${ }^{2}$ Nerlich (1979, 2010) is staunch advocate of the explanatory role of the geometry of spacetime, realistically construed. He classifies the role of physical geometry in such explanations as non-causal, but, on certain plausible understandings of causation (e.g., Lewis, 2000), it does count as causal (see also Mellor, 1980).

${ }^{27}$ The genesis of Einstein's general theory has been subject to extensive historical and philosophical scrutiny. For an excellent introduction to the topic, see Janssen (2008).
} 
spacetime, as encoded in the energy momentum tensor, $T_{a b}{ }^{28}$

$$
G_{a b}=8 \pi T_{a b} .
$$

One goal above all others guided Einstein's search: the generalization of the 'special' principle of relativity to a principle that upheld the equivalence of frames of reference in arbitrary states of relative motion. In this, Einstein was motivated by the belief that the role of primitive inertial structure in explaining phenomena in both Newtonian physics and in special relativity was "epistemologically suspect": real effects, he believed, should be traceable to observable, material causes.

In the early stages of his search, Einstein had a crucial insight, which he thought would play a key role in the implementation of a generalised relativity principle. According to his Principle of Equivalence, an inertial frame in which there is a uniform gravitational field is physically equivalent to a uniformly accelerating frame in which there is no gravitational field. It is not hard to see why one might take this principle to extend the principle of relativity from uniform motion to uniform acceleration 29

When Einstein first published the field equations of GR, he believed that their general covariance ensured that they implemented a general principle of relativity. Since smooth but otherwise arbitrary coordinate transformations include transformations between coordinate systems adapted to frames in arbitrary relative motion, it might seem that there can be no privileged frames of reference in a generally covariant theory. Almost immediately, Kretschmann (1917) pointed out that this cannot be correct, arguing that it should be possible to recast any physical theory in generally covariant form.

Compared to its predecessors, GR is without doubt a very special theory. But one will do more justice to its conceptual novelty, not less, by seeking as much common ground with previous theories as possible. We have already met generally covariant formulations of Newtonian and specially relativistic physics. As these examples attest, Kretschmann's instincts were sound and it is now well understood that the general covariance of GR does not implement a general principle of relativity. In fact, GR arguably includes privileged frames of reference in much the same way as pre-relativistic theories. ${ }^{30}$

\footnotetext{
${ }^{28} G_{a b}:=R_{a b}-1 / 2 R g_{a b}$, where $R_{a b}$ (the Ricci tensor) and $R$ (the Riemann curvature scalar) are both measures of curvature. $g_{a b}$ is the metric tensor and encodes all facts about the spatiotemporal distances between spacetime points. $R_{a b}$ and $R$ are officially defined in terms of the Riemann tensor, itself defined in terms of the connection $\nabla_{a}$. However, since we are considering the unique torsionfree, metric-compatible connection, we can view these quantities as defined in terms of the metric and, indeed, they can be given natural geometric interpretations directly in terms of spacetime distances. $T_{a b}$ encodes the net energy, stress and momentum densities associated with the material fields in spacetime.

${ }^{29}$ For a critical discussion of Einstein's various formulations of the principle, see Norton (1985).

${ }^{30}$ I should note that some still hold out against this orthodoxy (e.g. Dieks, 2006).
} 
It is illuminating to consider a concrete example. Compare the local spacetime formulation of specially relativistic electromagnetism to its generally relativistic version. Models of the former are of the form $\left\langle M, \eta_{a b}, F_{a b}, J^{a}\right\rangle$, where $\eta_{a b}$ encodes the Minkowski spacetime distances between the points of $M$, the covariant tensor field $F_{a b}$ represents the electromagnetic field and the vector field $J^{a}$ represents the charge current density. The dynamically possible models can be picked out via the coordinate-free form of Maxwell's equations:

$$
\begin{aligned}
\nabla_{a} F^{a b} & =-4 \pi J^{b} \\
\nabla_{[a} F_{b c]} & =\mathbf{0}
\end{aligned}
$$

The only difference between this theory and the generally relativistic theory is that, in the latter, the Minkowski metric $\eta_{a b}$ is replaced by a variably curved Lorentzian metric field, $g_{a b}$. Maxwell's equations remain as constraints on the DPMs of the GR version, and the same relativistic version of Newton's second law (which is formally identical to the non-relativistic version-equation ( $\mathrm{N}_{2}$ - given on page 13 above) holds in both theories ${ }^{31}$ But, whereas the specially relativistic metric was stipulated to be flat, to be physically possible according to GR, combinations of $g_{a b}, F_{a b}$ and $J^{a}$ must now obey the EFEs.

In the case of SR, the standard, Lorentz-invariant form of Maxwell's equations are recovered by choosing inertial coordinate systems adapted to the spacetime distances $\sqrt{32}$ The only reason that the same cannot be said of GR is that the pattern of spacetime distances catalogued by $g_{a b}$ does not allow for global coordinate systems that encode them. One can, however, always chose a coordinate system that is optimally adapted the spacetime distances in the infinitesimal neighbourhood of any point $p \in M$. In any coordinate system in which, at $p, g_{\mu \nu}=\operatorname{diag}(1,-1,-1,-1)$ and $g_{\mu v, \gamma}=0$, the laws governing matter fields will take their standard Lorentzinvariant form at $p$.

It is in terms of this strong equivalence principle that the phenomena that figure in Einstein's original principle are nowadays understood. ${ }^{33}$ From a modern perspective, apparent "gravitational fields" have precisely the same status as the potentials that give rise to the centrifugal and coriolis forces in Newtonian physics: they correspond to pseudo forces that are artefacts of the failure of the relevant coordinate systems to be fully adapted to the true geometry of spacetime. The "force" that holds us on the surface of the Earth and the "force" pinning the astronaut to the floor of the

\footnotetext{
${ }^{31}$ In this case, the four-force on a particle with charge $q$ and four-velocity $\xi^{a}$ is given by $q F^{a}{ }_{b} \xi^{b}$ and the equation is simply the coordinate-free version of the Lorentz force law.

${ }^{32}$ For example, the components of $F_{a b}$ relative to an inertial coordinate system are $F_{0 i}=-E_{i}, F_{i j}=$ $\epsilon_{i j k} B_{k}$, where $E_{i}$ and $B_{i}$ are the components of the electric and magnetic three-vector fields in that frame.

$3:$ Ehlers $(1973 b, 18)$; see Brown $2005,169-72)$ for a recent discussion.
} 
accelerating rocket ship are literally of one and the same kind. In GR, gravitational phenomena are not understood as resulting from the action of forces ${ }^{34}$

\section{Reasons to be a Relationalist?}

We have seen that substantivalism is recommended by a rather straightforward realist interpretation of our best physics. This physics presupposes geometrical structure that it is natural to interpret as primitive and physically instantiated in an entity ontologically independent of matter. Although I have only considered non-quantum physics explicitly, the claim is equally true of both non-relativistic and relativistic quantum theories (Weinstein, 2001). One might therefore wonder: why even try to be a relationalist? For some, the Hole Argument, discussed below in Section 7 , is the major reason to seek an alternative to substantivalism. Here I wish to review some other antisubstantivalist themes that have motivated relationalists. The conclusion will be that the only strong consideration in favour of relationalism is Ockham's razor: if a plausible relational interpretation of empirically adequate physics can be devised, then the standard reasons for postulating the substantivalist's additional ontology are undermined.

\subsection{A Failure of Rationality?}

In Section 6.2 I review Julian Barbour's approach to dynamics. According to Barbour, there is something "irrational" about standard Newtonian mechanics. Barbour sets up the problem in terms of the data required for the Newtonian initial value problem: given the equations of a Newtonian theory, what quantities must be specified at an instant in order to fix a solution? Following Poincaré, Barbour emphasises that the natural relational data-instantaneous relative distances and their first derivativesare almost but not quite enough. In addition, three further parameters, which specify the magnitude and direction of the angular momentum of the entire system,

\footnotetext{
${ }^{34}$ Some authors favour talk of "tidal forces" or state that there is a real "gravitational field" just where the Riemann tensor is non-zero (e.g. Synge, 1960, IX). As far as I can see, this is simply a misleading way of talking about spacetime curvature and (typically) nothing of conceptual substance is intended by it. For a discussion of some of the pros and cons of identifying various geometrical structures with the "gravitational field", see Lehmkuhl (2008, 91-8). Lehmkuhl regards the metric $g_{a b}$ as the best candidate. My own view is that consideration of the Newtonian limit (e.g. Misner et al. 1973. 445-6) favours a candidate not on his list, viz. deviation of the metric from flatness: $h_{a b}$, where $g_{a b}=\eta_{a b}+h_{a b}$. That this split is not precisely defined and does not correspond to anything fundamental in classical GR underscores the point that, in GR, talk of the "gravitational field" is at best unhelpful and at worst confused. The distinction between background geometry and the graviton modes of the quantum field propagating against that geometry is fundamental to perturbative string theory, but this is a feature that one might hope will not survive in a more fundamental "background-independent" formulation.
} 
are needed ${ }^{35}$ Barbour claims that there is something odd about this fact (see, e.g., Barbour, 2011, \$2.2).

Fixing the Euclidean relative distances between $N$ particles and their rates of change requires the specification of $6 N-12$ numbers; $6 N$ numbers are required to specify their positions and velocities in absolute space. The Newtonian initial value problem, however, requires $6 N-9$ numbers. Six of the $6 N$ numbers that specify the particles' absolute positions and velocities can be thought of as specifying the orientation of the system as a whole and the position of its centre of mass. States that differ solely in terms of these quantities only differ non-qualitatively, in terms of which particular points of space are related to the material system in (qualitative) ways common to both situations; the structural pattern of relations between space and matter is shared by both states. Even if such differences are to be regarded as real differences (something to be questioned in Section 7), Newtonian physics should not be expected to take them into account (pace Barbour, 1999, 83). It does not, after all, single out particular points of space by name. The remaining three parameters can be thought of as specifying the absolute velocity of the centre of mass. As we have seen, while the Newtonian substantivalist regards this as a genuinely qualitative matter, the spacetime substantivalist does away with these quantities by embracing neo-Newtonian spacetime. Arguably, $6 N-9$ numbers is precisely what the spacetime substantivalist should expect to be needed as Newtonian initial data.

What, then, is "irrational" about Newtonian mechanics? In Barbour's view it fails to be maximally predictive: relative to the Leibnizian data, there is an apparent breakdown of determinism, which is only restored by specifying the global angular momentum. At one level, this simply amounts to a prejudice in favour of the relational quantities over the Newtonian ones. For Barbour, this preference is based on the fact that it is the relational quantities that are "directly observable" (see, e.g., Barbour. 2010, 1280) but (i) direct observability is an extreme criterion for determining ontological commitment and (ii) instantaneous relative distances are not directly observable ${ }^{36}$ One can still agree with Barbour's less ambitious point. Without agreeing that there is something inherently objectionable about standard Newtonian theory, one might nevertheless prefer a theory that does as well (is at least as explanatory etc.) but with fewer resources. Barbour's observations about initial data highlight that, if an adequate relational theory of the envisaged type can

\footnotetext{
${ }^{35}$ Instantaneous relative distances and their first derivatives are the natural Leibnizian relational data. As reviewed in Section 6.2. Barbour's preferred framework for understanding classical mechanics also dispenses with a primitive temporal metric and an absolute length scale. With respect to these more frugal initial data, five, not three, additional numbers are needed. See Barbour (2011. $\$ 2.2)$.

${ }^{36}$ Nor are distance ratios, Barbour's preferred relational quantities. For an illuminating discussion of how instantaneous quantities are detected only indirectly, in measurements that necessarily take finite time, see Stein (1991, 157).
} 
be found, it will be more predictive, because its initial data form a proper subset of those of the Newtonian theory.

\subsection{The Spacetime Explanation of Inertia}

In Section 6.3 I consider the dynamical approach to special relativity, defended in Brown (2005) and Brown and Pooley (2006). I noted above that substantivalists view the postulated spacetime geometry as explanatory. Brown is suspicious of this doctrine. In assessing it, the putative explanatory roles of affine structure and of metric structure should be treated separately. Here I only consider the former; the latter is discussed in Section 6.3.

The idea that affine structure plays a quasi-causal role in explaining the motions of bodies figures significantly in Einstein's criticism of Newtonian mechanics and SR and in his subsequent understanding of GR. Consider Einstein's example from his early review paper on GR, of two fluid bodies separated by a great distance and in relative rotation about the line joining their centres. The two are of the same size, shape and nature except that one body is spherical whereas as the other is oblate. In Newtonian mechanics the explanation of this difference is that the oblate body, but not the spherical body, is rotating with respect to the inertial frames: the absolute rotation of the oblate body causes its oblateness. Einstein labels the Newtonian spacetime structure with respect to which such rotation is defined as a "merely factitious cause" of the difference; he held that a genuine explanation should instead cite another "observable fact of experience" (Einstein, 1916, 112-3). He initially maintained that this requirement was met in GR because he believed that the theory satisfied what he later called Mach's Principle (Einstein, 1918, 241-2): if the metric field $g_{a b}$ were fully determined by the distribution of matter throughout the universe, then the difference between the inertial behaviour of the two bodies would be traceable to differences in their relations to distant (observable) masses.

As Einstein soon recognised, GR does not satisfy Mach's Principle so defined. ${ }^{37}$ Inertial structure, as encoded in $g_{a b}$, is influenced but not determined by the matter content of spacetime ${ }^{38}$ Einstein ceased to regard the field describing inertial structure as having a secondary status relative to ponderable matter. By 1921, the objection to Newtonian absolute space was no longer that it was invisible. Instead

\footnotetext{
${ }^{37}$ De Sitter first pointed out to Einstein that, in addition to specification of $T_{a b}$, one needs to specify boundary conditions at infinity in order to determine $g_{a b}$. This prompted Einstein to search for spatially compact solutions to the EFEs and to introduce the cosmological constant to allow for a static, spatially closed universe. This in turn led de Sitter to the discovery of the de Sitter universe: a spatially compact vacuum solution to the modified EFEs. See Janssen (2008, \$5) for a summary of this episode and for further references.

${ }^{38} \mathrm{It}$ is also worth stressing that the stress-energy properties of matter, as encoded in $T_{a b}$, cannot even be defined independently of $g_{a b}$; see Lehmkuhl (2011).
} 
the fact that it acted without being acted upon was held up as problematic; a 'defect' not shared by the spacetimes of GR (Einstein, 1922, 61-2). ${ }^{39}$ At around the same time, Weyl advocated a similar conception of the role of inertial structure (he called it the "guiding field"), which he regarded as "physically real" in both pre-relativistic physics and in GR (Weyl, 1922, \$27).

What does Brown object to in this picture? Consider the "conspiracy of inertia": the relatively simple case of the force-free motions of a collection of Newtonian particles (Brown, 2005, 15, 141). Despite the fact that, ex hypothesi, the particles are not influencing one another, they move in a highly coordinated way: there are spacetime coordinate systems with respect to which all the trajectories are straight lines.$^{40}$ As we have seen, the coordinate-free geometrical description of this state of affairs regards the trajectories as coinciding with the straight lines of an affine connection on spacetime, the flatness of which allows for the global inertial coordinate systems in terms of which the phenomenon was stated. For the substantivalist, inertial structure is an element of reality that exists independently of the particles and their motions. On this view, aspects of the geometry play a role in explaining the phenomenon because, as stated in the substantivalist version of Newton's first law, the particles' trajectories are constrained as a matter of physical necessity to be aligned with features of this realistically construed geometry. For Brown, this is merely verbal pseudo-explanation. His preferred point of view reverses the arrow of explanation: the geometry is just a codification of the phenomenon, which (in pre-relativistic physics) must be taken as primitive.

If the only role of inertial structure was to explain pure inertial motion, Brown's complaint against the substantivalist would have some intuitive force; a flat affine connection could be thought of as a rather direct codification of the regularities manifest in the phenomena via the "coordinative definition" of spacetime geodesics as the trajectories of force-free bodies ${ }^{41}$ Explaining inertial motion, though, is

\footnotetext{
${ }^{39}$ The idea that something should be capable of acting if and only if it can also be affected by those things that it can influence is known as the action-reaction principle (see Anandan and Brown, 1995. for a discussion).

${ }^{40}$ I.e., there are coordinate systems with respect to which the particles' spatial coordinates are linear functions of their time coordinates. In Brown's view, "anyone who is not amazed by this conspiracy has not understood it" (Brown. 2005 15).

${ }^{41}$ The idea that spacetime geodesics are defined as the trajectories of force-free bodies is defended by DiSalle (1995, 327), whom Brown quotes approvingly. Elsewhere Brown, ostensibly to make a point against the substantivalist explanation of inertia, stresses that the principle that the trajectories of force-free bodies are geodesics in fact has limited validity in GR (Brown, 2005, 141, see also 161-8). What this observation in fact undermines is a relationalist approach to spacetime geometry that tries to define geodesics in terms of "basic physical laws" (DiSalle, 1995. 325). More recently, DiSalle makes clear that he differs from the logical positivists in not regarding the coordination of geodesics with free-fall trajectories as a matter of arbitrary stipulation. Instead it is said to be "a kind of discovery, at once physical and mathematical, that...the only objectively distinguishable state of motion corresponds to the only geometrically distinctive path in a generally covariant geometry"
} 
not the real purpose of inertial structure. Inertial structure figures centrally in the explanation of noninertial motion. In contrast to the force-free case, the sequence of relative distances between interacting particles manifest over time in a Newtonian universe displays no obvious regularity. It is a rather remarkable fact that, by postulating a highly symmetric geometrical structure in terms of which the motions of individual particles are to be understood, one can provide an elegant explanation, in terms of simple force laws, of the complicated and irregular history of relational quantities. Anyone who is not amazed by this conspiracy has not understood it. Since the postulated deep structure is not manifest in the surface phenomena it seems genuinely explanatory and not a mere codification. It is from an application of standard inference-to-the-best-explanation reasoning to this type of scenario that substantivalism gets its real support 42

Brown suggests that, around 1927, Einstein ceased to assign a quasi-casual role to spacetime in determining the inertial trajectories of bodies (Brown, 2005, 161). The alleged reason is that, at this time, Einstein came to recognise that the principle of inertia does not need to be postulated as a basic law in GR; it is instead a theorem. $4^{43}$ In Brown's view this fact undermines the idea that spacetime structure "in and of itself" acts "directly" on force-free bodes because it shows that, in GR, when such bodies undergo geodesic motion, "such motion is ultimately due to the way the Einstein field $g_{\mu \nu}$ couples to matter, as determined by the field equations" (Brown. 2005. 162-3). Brown's picture is, then, that the relationship of the motions of forcefree bodies to inertial structure in GR is radically different to this relationship in Newtonian physics and SR. In the latter theories, inertial structure is a mere codification of basic, mysterious inertial behaviour. In the former, it receives a dynamical explanation via the coupling of matter fields to the metric, as described by the EFEs.

The substantivalist should not be especially troubled by Brown's claim, for it concedes that, in our best (classical) theory of spacetime, the metric structure of spacetime is a primitive element of reality that plays a role in determining the inertial behaviour of bodies. Even so, there are reasons to doubt that the contrast between GR and pre-relativistic theories can really bear the weight Brown demands of it. The derivation of geodesic motion from the EFEs basically involves two steps. First, one notes that the EFEs imply the vanishing of the covariant divergence of the

(DiSalle 2006, 131-2). Nothing in the substantivalist's metaphysics is inconsistent with this position; it is less clear what other metaphysical views are compatible with it. DiSalle does not share the substantivalist's and relationalist's preoccupation with ontological questions but nor does he offer reasons to see such questions as illegitimate.

${ }^{42}$ Note that Einstein had in mind descriptions of interacting systems in different states of acceleration, and not simple inertial motion, when he claimed "that something real has to be conceived as the cause for the preference of an inertial system over a noninertial system" (Einstein, 1924, 16).

${ }^{43}$ See Malament 2010) for a critical discussion of this result. 
stress energy tensor: $\nabla_{a} T^{a b}=\mathbf{0}$. Secondly, one makes various assumptions about the nature of the stress-energy tensor to be associated with a force-free particle that, together with the vanishing of the divergence of stress-energy, can be shown to entail that the particle's trajectory is a geodesic. Now, the second step of this derivation is as applicable in SR as in GR ${ }^{44}$ What difference there is between the theories must therefore concern the status of the conservation principle, $\nabla_{a} T^{a b}=\mathbf{0}$. This equation, of course, also holds in SR. Further, while in SR it cannot be derived from the gravitational field equations (there are none), it is a consequence of the matter field equations (as it is in GR also)

In sum, geodesic motion is arguably as much a theorem in SR as it is in GR. An alternative perspective to Brown's is that the "dynamical coupling" of matter fields to inertial structure is in essential respects the same in GR and pre-relativistic theories. It is true that the geodesic theorem also demonstrates that it has limited validity in GR but not in SR (recall footnote 41). But the reason why, e.g., rotating bodies do not deviate from geodesics in SR is not that the relationship between realistically-construed inertial structure and matter is radically different in this theory to that in GR. The reason is simply that, in SR, there is no curvature to which rotating bodies might couple ${ }^{46}$

We have yet to meet a decisive reason to look for an alternative to substantivalism. Huggett, whose "regularity account" of relational spacetime I consider in Section 6.3. states that his reasons for advocating relationalism are the usual ones: "worries about 'Leibniz shifts' and considerations of ontological parsimony that militate against the introduction of bizarre non-material substances" (Huggett, 2006, 41). The kinematic shift has already been dealt with; other Leibniz shifts are discussed in Section 7 . It is not clear why Huggett regards spacetime as "bizarre". In a pre-relativistic context, one feature of spacetime that might seem odd is its failure, emphasised by Einstein, to obey the action-reaction principle. As we have seen, however, if this is a failing, it is not one shared by the spacetime of GR. That leaves considerations of ontological parsimony: other things being equal, a relationalist interpretation of physics might seem preferable to substantivalism because it makes do with fewer metaphysical commitments. The question is: are other things equal? It is time to examine some

\footnotetext{
${ }^{44} \mathrm{~A}$ closely parallel derivation is also possible in the geometrized form of Newtonian gravity; see Weatherall $(2011 a b)$. This might be taken to further undermine the claim that only in GR is inertia explained.

${ }^{45}$ See, e.g., Trautman $(1962,180-1)$.

${ }^{46}$ Brown's thesis that inertia receives a dynamical explanation only in GR has recently been defended by Sus (2011). Sus emphasises that in GR the metric is a genuinely dynamical entity and that one can derive $\nabla_{a} T^{a b}=\mathbf{0}$ from the very equations that govern the metric's behaviour. In contrast, $\mathrm{SR}$, as standardly conceived, involves fixed inertial structure whose properties are postulated by fiat. However, this difference between the theories is compatible with the theories agreeing on the fundamental reasons why force-free bodies are related to inertial structure in just the way they are.
} 
concrete relationalist proposals.

\section{Three Varieties of Relationalism}

Call the objects to whose existence a theory is committed the ontology of the theory. Call the range of (primitive) distinctions that a theory is able to express via its (primitive) predicates and terms-roughly, the set of (primitive) properties and relations to which the theory is committed-the theory's (primitive) ideology ${ }^{47}$ In these terms, the problem faced by the Leibnizian relationalist is that classical mechanics employs an ideology that appears to presuppose a substantivalist ontology. Inertial structure is naturally understood in terms of relations that hold of spacetime points; it cannot be understood (straightforwardly) in terms of properties and relations that are instantiated only by material objects. On the other hand, bona fide relationalist ideology appears to be too impoverished a basis for an empirically successful alternative to Newtonian theory.

This means that there are two obvious strategies open to relationalists. On the first, they can attempt to expand their ideology so that it underwrites the same physical distinctions as substantivalist inertial structure but in a way compatible with a relationalist ontology. To complete this programme, the relationalist then needs to re-construe standard Newtonian theory in terms of these new relationalist quantities ${ }^{48}$ Variants of this strategy are the topic of Section 6.1. On the second strategy, the relationalist seeks an alternative theory to Newtonian mechanics that employs only traditional relationalist quantities but is, although empirically distinct from Newtonian theory, nonetheless empirically adequate. Since the inertial frames are empirically determinable (in our neighbourhood of this universe), such a theory still needs to account for them, at least as a feature of solutions that could serve as models of the actual world. Unlike theories that result from the first strategy, however, it will not construe them as encoding primitive spatiotemporal properties and relations. The most promising version of this approach is reviewed in Section 6.2

These courses of action correspond closely to the first two of three options identified by Nick Huggett (1999). He sees Newton's globes thought experiment as illustrating that no theory has the following three characteristics. (i) Its spatiotemporal ideology is restricted to Leibnizian relations; (ii) its dynamically allowed histories of such relations are exactly those predicted by Newtonian theory and; (iii) inertial effects supervene on the specified spatiotemporal relations between bodies. Strate-

\footnotetext{
${ }^{47}$ The terminology is Quine's, who characterises a theory's ontology as "the objects over which the bound variables of the theory should be construed as ranging in order that the statements affirmed in the theory be true" (Quine, 1951, 11).

${ }^{48}$ The need for this second step is emphasised by Earman 1989, 128), though not in precisely these terms.
} 
gies (1) and (2) correspond to relinquishing (i) and (ii) respectively. But, Huggett observes, the relationalist might try to retain (i) and (ii) by dropping requirement (iii) (Huggett, 1999, 22-3). This amounts to a third, non-obvious strategy: don't change the theory and don't add to the ideology and yet somehow be a relationalist. This type of "have-it-all" relationalism is the topic of Section 6.3 .

\subsection{Enriched Relationalism}

\subsubsection{Classical Mechanics}

Part of the substantivalist's response to the kinematic shift argument involved replacing persisting space with spacetime. If relationalists likewise adopt a fourdimensional perspective, a number of options richer than Leibnizian relationalism become available. The most straightforward is Newtonian relationalism (Maudlin. 1993, 187). Whereas the Leibnizian relationalist posits spatial relations that hold only between simultaneous material events, the Newtonian relationalist simply posits that all material point events stand in spatial distance relations ${ }^{49}$

If we impose the natural constraints, the embedding of a Newtonian relational history into neo-Newtonian spacetime is fixed up to Galilean transformations. ${ }^{50}$ Whether this by itself entitles the relationalist to exploit the full resources of Newtonian dynamics (as Maudlin thinks; 1993, 192-3) need not be resolved, for the Newtonian relationalist can also interpret the dynamical laws directly in terms of relationalist quantities. For example, the absolute velocity of particle $i$ at time $t$ is just the limit, as $\delta t$ goes to zero, of the directed distance between the instantaneous stage of $i$ at $t$ and its instantaneous stage at $t+\delta t$ divided by $\delta t$. The Newtonian relationalist therefore has available a relational understanding of the very quantities that feature in the standard form of Newtonian laws expressed with respect to an inertial frame.

Unfortunately, Newtonian relationalism, like the Newtonian substantivalism from which its ideology is plundered, is vulnerable to the kinematic shift argument. ${ }^{51}$

\footnotetext{
${ }^{49}$ Related versions of relationalism, according to which absolute velocity (or even absolute position) is interpreted as a primitive, monadic property of particles, have been discussed by Horwich (1978, 403) and Friedman (1983, 235) (see, also, Teller 1987). In addition to being less natural than the form of Newtonian relationalism identified by Maudlin, they are vulnerable (like Newtonian relationalism) to the kinematic shift argument. The absolute position version is also vulnerable to the static shift argument mentioned in Section 7

${ }^{50}$ The obvious constraints are that the embedding respects the temporal separation between material events and that there is a single congruence of inertial geodesics such that, for any two material events $e_{1}, e_{2}$ located on geodesics from the congruence $v_{1}, v_{2}$, the Newtonian relational distance between $e_{1}$ and $e_{2}$ equals the (constant) spatial distance between simultaneous points of $v_{1}$ and $v_{2}$.

${ }^{51}$ This is the principal inadequacy of Newtonian relationalism that Maudlin identifies $(1993,193)$. Friedman (1983. 235) makes the same criticism of the postulation of a primitive property of 'absolute
} 
Since absolute velocities are unobservable, the Newtonian relationalist is committed to physically real distinctions that are in principle empirically inaccessible. The Newtonian relationalist must accept that, while only one inertial frame discloses the true Newtonian relational distances, that frame is forever beyond our grasp.

The spacetime substantivalist solves the kinematic shift problem by replacing Newtonian with neo-Newtonian spacetime. There is an obvious relational analogue of this move: since neo-Newtonian spacetime's inertial structure is equivalent to a relation of collinearity between triples of spacetime points, the relationalist can add to their ideology a three-place relation of collinearity between material events. The neo-Newtonian relationalist claims that, for three non-simultaneous events $e_{1}, e_{2}, e_{3}$, the relation $\operatorname{col}\left(e_{1}, e_{2}, e_{3}\right)$ holds just if, from the substantivalist perspective, $e_{1}, e_{2}$ and $e_{3}$ lie on a single inertial trajectory ${ }^{52}$

The move solves the kinematic shift problem, but only at the cost of leaving the relationalist's ideology too impoverished to fix the embedding of a relational history into neo-Newtonian spacetime. An example of Maudlin's nicely illustrates the point:

[C]onsider two particles in a neo-Newtonian spacetime that are uniformly rotating about their common center of mass. Until the first rotation is complete, no triple of occupied event locations are collinear. Even after any number of rotations, the collinearity relations among occupied points will be consistent with any periodic rotation, uniform or nonuniform. (Maudlin, 1993, 194)

One cannot, therefore, interpret the spacetime coordinates in which the dynamical laws take their standard form as just those coordinates adapted to the neoNewtonian relationalist's ideology; if the relationalist ontology is sufficiently sparse, this ideology underdetermines the inertial frames. ${ }^{53}$

This kinematical underdetermination is not necessarily fatal to neo-Newtonian relationalism. It means that the neo-Newtonian relationalist cannot simply lay claim to standard Galilean-invariant dynamics. On the strategy we are considering,

velocity'.

${ }^{52}$ Maudlin restricts the extension of $c o l$ to non-simultaneous events, but there is no reason why mutually simultaneous events should not be included, with $\operatorname{col}\left(e_{1}, e_{2}, e_{3}\right)$ holding just if the sum of the distances between two of the pairs of events equals the distance between the third pair.

${ }^{53} \mathrm{~A}$ similar example involving Minkowski spacetime is discussed by Mundy (1986), Catton and Solomon (1988) and Earman (1989, 168-9). The relations of spacelike separation, lightlike separation and timelike separation determine the structure of Minkowski spacetime up to an overall scale factor. However, these relations instantiated between material events need not fix their embedding into Minkowski spacetime up to Poincaré transformations. The examples discussed by Mundy et al. involve a small finite number of events, but the problem generalises to certain configurations of continuum many. E.g., consider two particles which move so that any two events from distinct trajectories are always spacelike (the events on each trajectory are all mutually timelike). We know that, as $t \rightarrow \pm \infty$, the particles must be accelerating in opposite directions, but not much more. 
however, the relationalist succeeds so long as they can identify, in a relationally respectable manner, a set of relational DPMs that correspond to the full set of Newtonian DPMs. Can the neo-Newtonian relationalist find dynamical laws expressed directly in terms of neo-Newtonian relations that achieve this?

As far as I know, no one has seriously attempted to construct such laws. Even so, one knows that any such laws will exhibit a particularly unattractive feature: they will not be expressible as differential equations that admit an initial value formulation. ${ }^{54}$ In standard Newtonian theory the specification of the instantaneous positions and velocities of the particles with respect to some inertial frame suffices, via the laws, to determine the particles' relative positions and motions at all times. Strictly speaking, what needs to be specified at an instant transcends the specification of the intrinsic state of that instant: in specifying velocities one is specifying quantities that are ultimately grounded in the pattern of the instantiation of collinearity relations between non-simultaneous spacetime points. But the relevant points lie in the infinitesimal neighbourhood of each instant and so can be used, via the usual limiting procedure, to define derivative quantities that are possessed at that instant. What Maudlin's example illustrates is that there can be finite stretches of time in a neo-Newtonian relational world such that no non-simultaneous triples of material events occurring during that time instantiate the collinearity relation. Indeed, this is a generic feature of neo-Newtonian relational worlds of point particles. The collinearity relation, therefore, cannot be used to define derivative quantities that can supplement the instantaneous data definable in terms of Leibnizian relations.

The problem faced by Newtonian relationalism suggested neo-Newtonian relationalism; the trouble faced by neo-Newtonian relationalism suggests another, rather desperate, relationalist manoeuvre. If the problem is the lack of appropriate instantaneous quantities, why not simply co-opt as primitive certain derivative instantaneous quantities available to the substantivalist? This, in essence, is how Sklar proposes the relationalist treat absolute accelerations; as intrinsic, primitive, time-varying properties possessed by particles at every instant of their trajectories (Sklar, 1974, 230). Huggett usefully dubs them Sklarations (Huggett, 1999, 27).

It is not obvious how the relationalist is supposed to use this additional ideology. Skow notes that simply supplementing Leibnizian relational initial data with Sklarations fails to fix a Newtonian history. Consider, for example, the following pair of two-particle solutions to Newton's theory of gravitation (Skow, 2007, 783-4). In one solution the two bodies follow circular orbits about their common centre of mass. In the second solution the particles travel in on parabolic paths from spatial infinity to sling-shot past each other before heading back out to infinity. Suppose that the distance of closest approach of the particles in the second solution matches

\footnotetext{
${ }^{54}$ This objection to neo-Newtonian relationalism, reported by Huggett (1999. 26), is again due to Maudlin.
} 
the constant separation of the particles in the first. Then, at the moment of closest approach, the second solution matches the first in terms of its Leibnizian initial data and absolute accelerations: the separation between the particles is the same, its rate of change is zero (it is the moment of closest approach) and, because accelerations due to gravity depend only on the masses of particles and the relative separation between them, the Sklarations are the same too.

In fact, this problem is generic. Precisely because, according to any Newtonian theory satisfying Newton's third law of motion, forces and hence absolute accelerations are functions only of the relative distances, they are effectively already included in the Leibnizian initial data. Thus every set of Leibnizian initial data "supplemented" with Sklarations will radically underdetermine the future evolution of any system of interacting Newtonian particles. As we saw in Section 5.1, this evolution depends on the overall angular momentum and the Leibnizian initial data, with or without Sklarations, does not tell us what this is.

Skow's assumption about the appropriate initial data for a theory employing Sklarations could be questioned. Why should the Sklar relationalist not include, say, the first time derivatives of Sklarations $\sqrt[5]{55}$ What the relationist really needs to provide are some relatively natural equations involving Sklarations that fix their theory's DPMs, for these will determine what the appropriate initial data are.

Sklar himself did not flesh out his proposal. Both Friedman $(1983,234)$ and Huggett $(1999,27)$ suggest that the Sklar relationist can simply utilise Newton's second law as expressed in arbitrary rigid Euclidean coordinate systems, i.e., coordinate systems adapted to the Leibnizian relational ideology. However, it is not at all straightforward how Sklarations are supposed to feature in such a formulation of Newton's second law ${ }^{56}$ More significantly, Friedman's and Huggett's attempt to reinterpret the standard equations in terms of Sklarations does not even get off the ground unless Sklarations are additionally constrained to be embeddable in spacetime as four-accelerations. But why should the instantiation of an allegedly primitive monadic property be constrained in this way as a matter of metaphysical necessity? Regarded as a kinematical constraint, the requirement is very fishy from

\footnotetext{
${ }^{55}$ Since these are functions of the $\dot{r}_{i j} \mathrm{~s}$, just as Sklarations are functions of the $r_{i j} \mathrm{~s}$, they would not actually be of any help either. The situation changes if higher derivatives are allowed.

${ }^{56}$ Friedman's expression of the law is $F^{i} / m=\ddot{x}^{i}+a^{i}+2 \dot{a}_{j i} \dot{x}^{j}+\ddot{a}_{k i} x^{k}$ (Friedman, 1983, 226, eqn 8; I have slightly altered the notation). $F^{i}$ is the $i$ th component of Newtonian (three-)force on the particle we are considering and $x^{i}(t)$ is the $i$ th component of its position vector with respect to some rigid Euclidean coordinate system. $a^{i}$ is the $i$ th component of the absolute acceleration of the origin of the coordinate system (i.e., the Sklaration that a hypothetical particle would have were it comoving with the coordinate origin). $\dot{a}_{j i}$ is the rotation of the coordinate system about its origin with respect to an inertial frame. Thus only the first of the three additional terms on the right-hand side of the equation is directly interpretable in terms of a Sklaration, and then only if we pick a coordinate system that happens to have a particle comoving with its origin. Crucially, we need to be told how to interpret the rotation pseudo-vector $\dot{a}_{j i}$ in terms of Sklarations.
} 
a relationalist perspective. The alternative is to view the constraint only as a restriction on the physically possible, i.e., as an additional "law of motion" governing the evolution of Sklarations (and constraining admissible initial data) ${ }^{57}$ Either way, a strong suspicion must remain that, in this guise, Sklarations do not constitute a genuine alternative to accelerations and the attendant substantivalist commitments they require.

The relationalist needs ideology weaker than Newtonian relations but richer than the neo-Newtonian's collinearity relation. In particular, ideology that is sufficiently richly instantiated in the neighbourhood of any instant is needed in order to avoid the initial value problem faced by the neo-Newtonian relationalist. Sklarations might have been expected to provide what was needed but, because the quantities are treated as primitive, their necessary connections with the relational states of the world at earlier and later times is severed. Putting these connections back in by hand looks like substantivalism by another name.

This suggests that to avoid the pitfalls of Sklarations the relationalist should look for ideology that is instantiated by some $n$-tuples of non-simultaneous events. And to avoid the pitfalls of neo-Newtonian relationalism, this ideology should be instantiated by $n$-tuples of non-simultaneous events in the infinitesimal neighbourhood of any instant. Further, any kinematic constraints on the possible instantiation of this ideology should be comprehensible independently of its interpretation, once appropriately embedded, in neo-Newtonian spacetime.

It is certainly possible to specify relational ideology that meets these requirements ${ }^{58}$ but at this point we should take a step back and recall the structure of the original dilemma posed by Galilean invariance. A dynamical symmetry group that was larger than the spacetime symmetry group leads to in-principle unobservable quantities; a spacetime symmetry group larger than the dynamical symmetry group requires a non-standard story about the privileged status of some dynamically preferred frames of reference (for they form a proper subset of those maximally adapted to the spacetime quantities). I have given the impression that, in the context of classical mechanics, the structures of neo-Newtonian spacetime get things just right, but in fact this is not the case: the dynamical symmetry group of Newtonian physics is in fact larger than the Galilei group.

In inertial frame coordinates, the field-theoretic form of Newton's law of gravi-

\footnotetext{
$5 \sqrt{\text { Mundy }}(1983,224)$ even interprets the Euclidean constraints on instantaneous distances similarly, so that his relationalist does not need a primitive notion of geometric possibility over and above that of physical possibility.

${ }^{58}$ I explore some of the options in Pooley (in preparation Ch. 3). As with Sklarations, the required "kinematical" constraints on the instantiation of such relations suggest that the proposals are really substantivalism in disguise.
} 
tation is expressed by the following equations:

$$
\begin{aligned}
\vec{F} & =-m \vec{\nabla} \phi \\
\nabla^{2} \phi & =4 \pi G \rho
\end{aligned}
$$

(Grav Force)

(Poisson)

where $\phi$ is the gravitational potential, $G$ is Newton's constant and $\rho$ is the mass density. In the coordinate-free notation of Section 4 , these equations become:

$$
\begin{aligned}
F^{a} & =-m h^{a b} \nabla_{b} \phi \\
h^{a b} \nabla_{a} \nabla_{b} \phi & =4 \pi G \rho .
\end{aligned}
$$

and the theory has models of the form $\left\langle M, t_{a b}, h^{a b}, \nabla_{a}, \rho, \phi\right\rangle$. The coordinatedependent equations are invariant under Galilean transformations, which are also dynamical symmetries in the model-theoretic sense, but these transformations do not exhaust the symmetries. Consider the Maxwell grouf ${ }^{59}$ of coordinate transformations:

$$
\begin{aligned}
\vec{x}^{\prime} & =\mathbf{R} \vec{x}+\vec{a}(t), \\
t^{\prime} & =t+d .
\end{aligned}
$$

Like those of the Leibniz group, they involve an arbitrary, time-dependent translation term, $\vec{a}(t)$. Like those of the Newton and Galilei groups, the rotation matrix $\mathbf{R}$ is not time-dependent. They therefore correspond to a spacetime structure that embodies an absolute standard of rotation but no general standard of acceleration. ${ }^{60}$

These transformations will also preserve the coordinate-dependent form of the equations of Newtonian gravitation, and map solutions to solutions, so long as the gravitational potential field is also transformed appropriately:

$$
\phi \mapsto \phi^{\prime}=\phi-\vec{x} \cdot \ddot{\vec{a}}+f(t),
$$

where $f(t)$ is an arbitrary time-dependent function that is constant on surfaces of simultaneity. It follows that, if $d$ is a diffeomorphism corresponding to such a transformation and $\left\langle M, t_{a b}, h^{a b}, \nabla_{a}, \rho, \phi\right\rangle$ is a model of the spacetime formulation of Newtonian gravity we are considering, then so is $\left\langle M, t_{a b}, h^{a b}, \nabla_{a}, d^{*} \rho, \phi^{\prime}\right\rangle$. This means that the neo-Newtonian substantivalist is in precisely the same kind of predicament as the substantivalist who advocated absolute space: their metaphysics grounds physical quantities (in this case absolute accelerations, rather than absolute velocities) that it is impossible in principle to detect. Here is another way to see the

\footnotetext{
${ }^{59}$ My terminology again follows Earman (1989, 31) and Bain (2004, 351). Ehlers (1973a. 78-9) discusses the group but leaves it unnamed.

${ }^{60}$ There is no canonical differential-geometric way of capturing this structure. Earman (1989, 32) resorts to an equivalence class of connections whose congruences of geodesics are non-rotating with respect to one another. Saunders $2012, \$ 7)$ offers an elegant characterisation of a similar but strictly weaker structure.
} 
problem. Since, for the type of diffeomorphism under consideration, $d^{*} t_{a b}=t_{a b}$ and $d^{*} h^{a b}=h^{a b}$, if $\mathcal{M}_{1}=\left\langle M, t_{a b}, h^{a b}, \nabla_{a}, \rho, \phi\right\rangle$ is a model of the theory, then so is $\mathcal{M}_{2}=\left\langle M, t_{a b}, h^{a b}, \nabla_{a}^{\prime}, \rho, \phi^{\prime}\right\rangle$, where $\nabla_{a}^{\prime}=\left(d^{-1}\right)^{\star} \nabla_{a}$. I.e., the laws and a given matter distribution $\rho$ fix the temporal and spatial metric structures, but they leave it underdetermined whether the combination of inertial structure and gravitational force is that given by $\left(\nabla_{a}, \phi\right)$ or by $\left(\nabla_{a}^{\prime}, \phi^{\prime}\right)$. And if we take the postulated inertial structures ontologically seriously, these differences correspond to qualitative differences. For example, in one model a given particle might be force-free and moving inertially; in the other it might be accelerated under a gravitational force ${ }^{61}$

A natural thought at this point is that $\mathcal{M}_{1}$ and $\mathcal{M}_{2}$ are merely mathematically distinct representations of the same physical possibility and that $\phi$ and $\nabla_{a}$ are gaugedependent quantities. But one cannot simply declare this so by fiat. One should also provide a characterisation of a gauge-invariant reality in terms of which the gauge dependent quantities can be understood. It turns out that the substantivalist can, indeed, do this. The solution is Newton-Cartan theory, a formulation of Newtonian gravitation first developed by Cartan and Friedrichs ${ }^{62}$ In this theory, just as in GR, gravitational phenomena are not the effects of forces. The flat inertial connection $\nabla_{a}$ is replaced by dynamical inertial structure $\nabla_{a}^{\mathrm{NC}}$ (in part) governed by the following generalisation of the coordinate-free form of Poisson's equation:

$$
R_{a b}^{\mathrm{NC}}=4 h G \rho t_{a b},
$$

$\left(\right.$ Poisson $\left._{\mathrm{NC}}\right)$

which relates the Ricci tensor $R_{a b}^{\mathrm{NC}}$ defined by $\nabla_{a}^{\mathrm{NC}}$ to the mass density. Our two models of Newtonian gravity set in neo-Newtonian spacetime, $\mathcal{M}_{1}$ and $\mathcal{M}_{2}$, correspond to a unique model of Newton-Cartan theory (up to isomorphism). Any given $\left(\nabla_{a}, \phi\right)$ pair that solves non-geometrized Newtonian gravity determines a unique dynamical connection but the converse is not true: a given Newton-Cartan connection can always be decomposed into a flat connection and a gravitational potential, but this decomposition is non-unique in a way that corresponds exactly to the underdetermination of gravitational theory set in neo-Newtonian spacetime. ${ }^{63}$

While the problem of the symmetries of Newtonian gravity and its substantivalist solution are relatively well-known, the fact that an enriched-ideology relationalist strategy can also be fruitfully pursued is far less appreciated. When canvassing enriched relationalist options earlier in the section, the operative assumption was that Newtonian dynamics was Galilean invariant. Now that the larger Maxwell

\footnotetext{
${ }^{61}$ This problem for Newtonian gravitation set in neo-Newtonian spacetime is well-known. For a related discussion in the philosophical literature, see, e.g., Friedman (1983, 95-7).

${ }^{62}$ This theory is presented as the solution to the problem faced by the Galilean substantivalist by Friedman (1983, 97-104; 120-4). See also Malament (1995), who presents it as a solution to a closely related problem raised by Norton (1993).

$6:$ Malament (2012, Ch. 4) reviews these results and Newton-Cartan theory more generally; see also Bain (2004).
} 
group has been recognised as a symmetry group, a reevaluation is needed. The equations of any $\mathrm{N}$-body Newtonian system whose force laws obey Newton's third law can be re-expressed as:

$$
\ddot{\vec{r}}_{i j}=\frac{1}{m_{i}} \sum_{k \neq i}^{N} \vec{F}_{k i}-\frac{1}{m_{j}} \sum_{k \neq j}^{N} \vec{F}_{k j}
$$

where $\vec{r}_{i j}:=\left(\vec{x}_{i}-\vec{x}_{j}\right)$ is the directed relative distance between particles $i$ and $j$ and $\vec{F}_{i j}$ is the force exerted by particle $i$ on particle $j$ (Hood, 1970; see Earman. 1989, 81, for discussion). For the time derivatives of $\vec{r}_{i j}$ to be well defined, the full inertial structure of neo-Newtonian spacetime is not required. All that is needed is a standard of rotation, i.e., exactly the spatiotemporal structure invariant under the Maxwell group. Since we are assuming Newton's third law is also satisfied, the only spatial dependence of $\vec{F}_{i j}$ will be on $\vec{r}_{i j}$. It follows that Equation (1) is invariant under the Maxwell group. The only ideology, in addition to Leibnizianrelational quantities, needed to ground a standard of rotation is the transtemporal comparison of the directions of the directed distances between material bodies. ${ }^{64}$ For example, the Maxwellian relationalist can postulate a primitive four-place relation $A$ on material events such that, when $\left(e_{1}, e_{2}\right)$ and $\left(e_{3}, e_{4}\right)$ are pairs of simultaneous events, $A\left(e_{1}, e_{2}, e_{3}, e_{3}\right)$ takes a value between 0 and $2 \pi$, to be interpreted as the angle between $\overrightarrow{e_{1} e_{2}}$ and $\overrightarrow{e_{3} e_{4}}$.

What this shows is that the full set of Newtonian solutions for a finite system of interacting particles can be given a bona fide relationalist interpretation (with or without Newtonian gravitation). With Earman (1989, 81), we should now ask whether the basic idea can generalise to field theory. Maxwellian relationalism for field configurations can easily be implemented using Barbour's best-matching machinery, discussed in Section 6.2 so field theory per se is not an obstacle. ${ }^{65}$ Barbour's machinery, however, is only applicable to spatially finite systems (or systems with appropriate spatial boundary conditions). In such "island universe" scenarios, the

\footnotetext{
${ }^{64}$ As far as I am aware, Simon Saunders was the first to stress that transtemporal comparison of directions are obviously compatible with relationalist ontology. Saunders (2012) is a recent discussion of related topics. I am grateful to him for discussion. Earman 1 1989.78-81) comes close to attributing the basic idea to James Clerk Maxwell, who, when discussing absolute rotation in Maxwell (1877. $\$ 104$ ), wrote: "in comparing one configuration of the system with another, we are able to draw a line in the final configuration parallel to a line in the original configuration." Earman's assessment is that "Maxwell's set of parallel directions is, of course, inertial structure, and in modern terms what he seems to be proposing is that neo-Newtonian spacetime is the appropriate arena for the scientific description of motion" (Earman, 1989. 80). However, it is clear that Maxwell here only assumes a standard of parallelism for spacelike lines which, as we have seen, does not require the full structure of neo-Newtonian spacetime. Perhaps Earman did not realised how apt his label Maxwellian spacetime is.

${ }^{65}$ One "best matches" instantaneous configurations only with respect to rigid translations and not, as Barbour does, by translations, rotations and dilations.
} 
Maxwellian invariance of dynamics does not trouble the neo-Newtonian substantivalist, for a preferred inertial connection can be identified via the condition that the total three-momentum of the whole system is constant. Underdetermination only genuinely arises for the neo-Newtonian substantivalist in Newtonian cosmology when we consider, e.g., infinite homogeneous matter distributions. As far as I know, no relationalist theory for such situations has been devised. The Maxwellian relationalist seems to be in the unfortunate position of having a solution applicable to those cases that are not genuine problems and no solution for the truly troubling cases.

In the context of field theory, there is one relatively easy way out for the relationalist. ${ }^{66}$ Recall that the troubles faced by the neo-Newtonian relationalist arose because, in a world of point particles, the three-place collinearity relation typically will not be instantiated by material events in the infinitesimal neighbourhood of a given material point. If the relationalist embraces a plenum, this problem goes away. In the context of Newtonian gravity, the relationalist can combine a material plenum with the insight of Newton-Cartan theory and postulate a primitive three-place collinearity relation on material events that holds of triples of material events in a physically possible world just if, in the corresponding substantivalist model, they lie on a geodesic of the substantivalist's dynamical affine connection. Such a Newton-Cartan relationalist still has work to do. The characterisation of the position just given made crucial reference to substantivalist models. Can the standard mathematical formalism of Newton-Cartan theory be independently understood in terms of such relational ideology? What are the material fields and why must they constitute a plenum? Similar questions recur in the context of relativistic physics, where fields are no longer optional extras. It is to relativity we now turn.

\subsubsection{Relativity}

In the context of classical mechanics, the relationalist who pursues the enrichedideology strategy is forced to be creative. Simply co-opting substantivalist ideology (by restricting the domain of possible instantiation to the material events) fails, primarily because of the relative sparseness of the relationalist's ontology in comparison to the substantivalist's plenum of spacetime points. In the context of SR, however, the flat-footed move works. Restricting the substantivalist's ideology-Minkowski spacetime distances-to material events fixes the embedding of a relational history into Minkowski spacetime (up to isomorphism). Further, Minkowski distances conceived of as relationalist ideology can be used to frame dynamical principles directly in relationalist terms ${ }^{67}$

\footnotetext{
${ }^{66}$ I am grateful to David Wallace for highlighting this possibility.

${ }^{67}$ The main discussions of a position of this sort are Earman 1989, 128-30) and Maudlin 1993 196-9).
} 
Central to relativistic mechanics (even if not to relativistic physics in general), is the idea that unaccelerated motion is default behaviour and that accelerations are due to forces. In order to lay claim to this picture, the Minkowski relationalist needs accounts of both accelerations and forces. In classical mechanics, forces were unproblematic for the relationalist (because they are functions of Leibnizian relational quantities); it was acceleration that proved troublesome. In relativity, the difficulties are reversed.

Consider the standard, coordinate-dependent forms of relativistic laws. The privileged class of coordinate systems relative to which these equations hold are simply those adapted to the Minkowski distance relations between material events (cf. page 17). Dynamically significant absolute acceleration, therefore, is simply acceleration relative to the coordinate systems adapted to the relationalist's spatiotemporal distances. In fact, the Minkowski relationalist can do better and give an intrinsic characterisation of acceleration. Recall that, in Minkowski spacetime, the inertial trajectories are not structure over and above the spatiotemporal distances; the straight line in spacetime between two temporally separated events is the path of maximal temporal distance. This means that a particle will be unaccelerated just if, for any temporally ordered points $p, q, r$ of its trajectory, $I(p, r)=I(p, q)+I(q, r)$ and, conversely, if $I(p, r)>I(p, q)+I(q, r)$, we know that the particle is accelerated between the points $p$ and $r($ Earman, 1989, 129). The four-acceleration of a trajectory at a point just is the intrinsic curvature of the trajectory at that point and so, as for curves in Euclidean space, one can define the acceleration of the particle (both its magnitude and its direction) in terms of such distances ${ }^{68}$

The Minkowski relationalist treatment of forces is less straightforward. The coordinate-free statement of the second law, $F^{a}=m \xi^{n} \nabla_{n} \xi^{a}$, is formally the same in classical mechanics and SR and, in both cases, the four-force, $F^{a}$, must be a spacelike vector. This formal identity, however, hides a crucial difference. In the neoNewtonian case, spacelike vectors lie in (i.e., are tangent to) surfaces of simultaneity. As a result neo-Newtonian four-forces can be defined in terms of Leibnizian spatial distances, which are intrinsic to such surfaces. In the Minkowski case, if one considers an arbitrary spacelike hyperplane and the accelerations of a number of interacting particles at the points where their trajectories intersect this plane, then, in general, none of these accelerations will be tangent to the hyperplane. It is no accident that in relativistic theories $F^{a}$ is standardly given as a local function of fields. ${ }^{69}$

\footnotetext{
${ }^{68} \mathrm{~A}$ treatment of acceleration along these lines can be found in Minkowski's original presentation (Minkowski, 1908, 85-6).

${ }^{69}$ Relativistic theories in which the four-force on a particle at a point is determined directly by the properties of other particles at other spacetime locations are not impossible; Feynman and Wheeler's version of electromagnetism is such a "pure particle" theory (Wheeler and Feynman, 1949). These theories, however, have various unwelcome features, and their empirical adequacy remains an open
} 
Some think that the need to invoke fields is a problem for the relationalist. On one (natural) interpretation, fields are simply assignments of properties or states to the points of spacetime (Field, 1985, 40). Such a view does indeed presuppose substantivalism, but there is an alternative available, and it is one that arguably fits more naturally the language employed by physicists. On this other view, the field itself is reified as a vast, spatiotemporally extended object in its own right ${ }^{70}$ Adopting this second conception of fields does not, by itself, amount to relationalism; many substantivalists will agree that at least some fields are extended objects in spacetime (rather than properties of spacetime) ${ }^{71}$ Taking a 'relational' view of a field also does not by itself commit one to the view that such a field could exist in the absence of spacetime, or have the very properties one's theory characterises it as having ( $c f$. footnote 38 ). The devil will be in the details.

Consider the simple case of a field, $\phi$, with just one degree of freedom per spacetime point. The relationalist wishes to view $\phi$ as an extended, physical entity rather than as an assignment of properties to spacetime. Since spacetime itself is supposed not to exist, this extended object cannot be characterized in terms of the spatiotemporal locations of the various field intensities. Instead, the relationalist should view the field as characterized by the infinite number of facts about the Minkowski distances between its pointlike parts; together these fully characterise the pattern of field intensities.72 These distances cannot (in practice) be specified directly. But there is nothing relationally improper about describing the field relative to a Lorentzian coordinate system so long as such a chart is thought of as a map directly from the field itself into $\mathbb{R}^{4}$ that encodes the Minkowski distances.

Consider, now, the substantivalist's presentation of a theory of such a field. The KPMs will be of the form $\left\langle M, \eta_{a b}, \phi\right\rangle$ and the DPMs will be picked out via an equation relating $\phi$ and $\eta_{a b}$. Suppose the relationalist's only way to identify dynamically possible field configurations was to use this machinery. Would they then be in the embarrassing position of relying on a substantivalist "fairy tale" without a proper explanation of why it works (Earman, 1989, 172)? It doesn't seem so. That $\phi$ is the only field in the model reified by the relationalist does not mean that $\eta_{a b}$ is a fiction. The substantivalist suggests that one understands fields as assigning various properties and relations. In this case, the relationalist agrees. They just

question; see Earman (1989, 155-8) for discussion.

${ }^{70}$ See Malament (1982, 532, fn 11), who is responding to Field's argument. Other clear expressions of this view can be found in Belot (1999, 45) and Rovelli (2001, 104).

${ }^{71}$ As the rest of this section illustrates, still less does the move trivialise the substantivalistrelationalist debate (pace Field, 1985, 41), although it does excuse the relationalist from replacing field theories with action-at-a-distance theories.

${ }^{72}$ Note that this constitutes an answer to Earman's challenge that the relationalist must provide a "direct characterization" of reality underlying the substantivalist's description of fields (Earman, 1989. 171). 
disagree about the subject of predication: for the substantivalist it is spacetime itself, for the relationalist it is the one substantival field of the model, $\phi$. Equations relating $\phi$ to the other fields then have a straightforward relationalist reading as claims about the allowed (geometrical) properties of $\phi$ itself.

So far I have only considered scalar fields. More complex fields can pose additional problems for the relationalist. Standard vector and tensor fields, for example, are not obviously conceptually independent of the structure of the manifold on which they are defined. Their degrees of freedom at a point are normally understood as taking values in the tangent space at that point (or in more complex spaces constructed in terms of it), which might appear to presuppose the differentiable structure of the manifold on which the fields are defined. In fact, even characterising scalar fields normally involves this manifold structure, for one is normally interested in smooth fields. In this case, however, it is clear how one can do away with reference to an independent manifold. What one requires (roughly speaking) is that the field's values vary smoothly as a function of the distances between its parts: fields themselves can have the structure of a differentiable manifold in virtue of these Minkowski distances. Vector and tensor fields, conceived of as substantival entities in their own right, will likewise have a manifold structure, but there is something suspiciously circular about taking the spaces in terms of which a field's degrees of freedom are defined to be themselves defined in terms of that field's own spatiotemporal extension. An alternative is to try to understand the degrees of freedom of some material fields in terms of their interactions with other fields whose relational credentials are not in doubt 73

The upshot is that the combination of Minkowski relationalism and a relational interpretation of fields is at least a going concern as an interpretation of SR. The final task for this section is to consider whether the picture can be adapted to GR. The strong similarities between SR and GR stressed in Section 4.3 might lead the relationalist to be optimistic. In fact, the move from flat to curved geometric structure, and the manner in which it features in GR, presents a formidable obstacle. Recall that the Minkowski relationalist does not reify the metric field $\eta_{a b}$. Instead this field is regarded as cataloguing primitive spatiotemporal distances that hold between the parts of bona fide material fields. At the level of kinematics, the generalisation of this to GR is straightforward. Minkowski distances are simply replaced by those of a curved semi-Riemannian geometry. A crucial consequence of this move is that the distances instantiated between material events need no longer fix (independently of the dynamical laws) all the facts about the geometry of spacetime. In particular, consider an "island universe" involving a matter distribution of finite spatial extent. The spatiotemporal distances instantiated in the history of the material world will

\footnotetext{
${ }^{73}$ See, for example, Malament $(2004$ \$3), where the tensorial properties of the electromagnetic field $F_{a b}$ are derived from assumptions about its action on charges.
} 
not fix the geometry of the empty spacetime regions beyond it.

This is not a problem of principle. After all, the relationalist will claim that there is literally nothing beyond the boundary of the material universe to instantiate one geometry rather than another. There is also no difficulty, in principle, with this type of relationalist regarding geometry as dynamical and as influenced by matter. For example, the laws of a relational theory could lay down how the network of spatiotemporal relations instantiated in some temporally thick slice through the material world determine (together with other dynamically relevant properties of matter) the pattern of spatiotemporal relations instantiated in earlier and later regions of the material universe. The particular difficulty GR poses for the envisaged relationalist involves the combination of these two factors. In GR the geometrical properties of the supposed non-entity beyond the material universe do make a dynamical difference. For example, the entire history of spatiotemporal distances instantiated in our island universe up to some time will not record whether a "gravitational wave" (i.e., a propagating ripple in the fabric of spacetime itself) is approaching from outside the system and will thus underdetermine the system's future evolution (Earman, 1989, 130; Maudlin, 1993, 199).

In response, the relationalist could rule out by fiat models with empty regions of spacetime. To do so, however, is not only to give up on the goal of empirical equivalence with standard theory; it is to impose a restriction that is arbitrary by the lights of the relationalist's own theoretical apparatus. The relationalist does not have problems with empty regions per se. What they have problems with is those regions having a determinate geometry that need not supervene on the properties of and relations between matter-filled regions and with the geometry of empty regions playing the dynamical role that GR assigns it. The better "relationalist" move is to treat the metric tensor as a "material" field in its own right, but then, since all parties affirm the existence of a substantival entity whose properties are characterised by $g_{a b}$, it is not clear what substantive issue remains. ${ }^{74}$

\subsection{Barbour's Machian Relationalism}

There are two straightforward relationalist responses to the mismatch between relationalist spacetime symmetries and the dynamical symmetries of Newtonian mechanics. The previous section covered one of these: enrich relationalist ideology in order to bring spacetime symmetries into line. This section investigates the other: change the dynamics in order to bring the dynamical symmetries into line. The most

\footnotetext{
${ }^{74}$ The relationalist can also question whether one should regard regions of zero field strength as regions where the material field literally does not exist. This might be the natural interpretation of fields that represent "dust" in models of GR, but it is at least controversial for, e.g., the electromagnetic field. The stipulation is yet more problematic when one moves to quantum field theory. I am grateful to David Wallace for pressing this point.
} 
thorough and successful development of this strategy is that of Julian Barbour and collaborators. The label Machian relationalism is appropriate for three reasons. First, it accords with Barbour's own terminology. He sees the requirement that a theory be maximally predictive with respect to relational initial data (in the sense discussed in Section 5.1) as a precise version of Mach's Principle and he takes his approach to dynamics to reveal that GR is in fact a Machian theory. Secondly, in the context of prerelativistic particle dynamics, the spacetime quantities Barbour takes as fundamental are even sparser than those of the Leibnizian relationalist: the Euclidean nature of the instantaneous relative distances between particles is accepted as primitive, but the temporal intervals between successive instantaneous configurations are not. In his critique of Newton, Mach (1901, 222-6) claimed that the question of whether a motion is in itself uniform is senseless, on the grounds that a motion can (allegedly) only be judged uniform relative to some other motion or material process..$^{75}$ Finally, Barbour's particle theories provide a concrete implementation of Mach's idea that the inertial properties of a body might be understood in terms of that body's relations to the rest of the bodies in the universe, rather than with respect to substantival spacetime structure (Mach, 1901, 231-5).

Up to this point I have presented the DPMs of a theory as singled out in terms of differential equations that must be everywhere satisfied within a model by its constituent fields and particle trajectories. In some formulations of dynamics, the DPMs are singled out in terms of their relations to other KPMs. Machian relational theories are most illuminatingly developed in this type of framework. Consider, in particular, the Lagrangian formulation of Newtonian mechanics. Central to this framework is a system's configuration space, $Q$, the points of which represent possible instantaneous states of the system. According to the substantivalist, such a state for an $\mathrm{N}$-particle system corresponds to a set of positions for each particle relative to some inertial frame. $Q$ is then $3 \mathrm{~N}$-dimensional. As the system evolves, the point in $Q$ representing the system's instantaneous state traces out a continuous curve. In Lagrangian mechanics, KPMs (i.e., metaphysically possible histories) are (monotonically rising) curves in the product space formed from $Q$ and a onedimensional space, $T$, representing time. The DPMs are those curves that extremize a particular functional of such histories (the action).

This framework can be adapted to Leibnizian and Machian relationalism in a straightforward way. First, since the relationalist's possible instantaneous states correspond to sets of inter-particle distances (rather than positions defined with respect to spacetime structure), the relationalist replaces $Q$ with the relative configuration space $Q_{\mathrm{RCS}}$. For $N$ particles, $Q_{\mathrm{RCS}}$ is $(3 N-6)$-dimensional. In fact, the relationalist

\footnotetext{
${ }^{75}$ For related reasons, Earman defines Machian spacetime to be spacetime with simultaneity structure and Euclidean metrical structure on its simultaneity surfaces but with no temporal metric (Earman 1989, 27-30).
} 
might be tempted to go further. Formulating dynamics in terms of $Q_{\mathrm{RCS}}$ involves treating transtemporal comparisons of length as primitive. Distinct curves in $Q_{\mathrm{RCS}}$ can correspond to exactly similar sequences of Euclidean configurations if some of the corresponding configurations represented in the two curves differ in overall size. This is not true in shape space $\left(Q_{\mathrm{sS}}\right)$, a configuration space of one less dimension that treats only the ratios of distances within configurations as physically meaningful.

Secondly, standard theory distinguishes histories that correspond to a single curve in configuration space being traced out at different rates with respect to the primitive temporal metric. The Machian relationalist, in contrast, will view each curve in configuration space as corresponding to exactly one possible history. They therefore dispense with $T$, the space encoding primitive temporal separations, in favour of a "timeless" formulation of dynamics in terms of configuration space alone. One way to do this is to equip the space with a metric. The DPMs are then picked out via a geodesic principle: physically possible histories correspond to paths in configuration space of extremal length relative to the metric.

The implementation of this second step can be achieved via a reinterpretation of Jacobi's Principle, part of the standard toolkit of Newtonian dynamics. ${ }^{76}$ The metric structure of three-dimensional physical space can be used to define a metric on $Q$ known as the kinetic metric: $d s_{\text {kin }}^{2}=\sum_{i} \frac{m_{i}}{2} d \vec{x}_{i} \cdot d \vec{x}_{i}$. Its geodesics correspond to histories of particles moving inertially. Dynamics is incorporated by multiplying $d s_{\text {kin }}^{2}$ by a conformal factor $F_{E}=\left(E-V\left(\vec{x}_{1}, \ldots, \vec{x}_{N}\right)\right)$. The geodesic principle is then:

$$
\begin{gathered}
\delta I=0, \quad I=\int \sqrt{F_{E}} d s_{\text {kin }}=2 \int d \lambda \sqrt{F_{E} T_{\text {kin }}}, \\
\text { where } \quad T_{\text {kin }}:=\frac{1}{2} \sum_{i}^{N} m_{i} \frac{d \vec{x}_{i}}{d \lambda} \cdot \frac{d \vec{x}_{i}}{d \lambda} .
\end{gathered}
$$

Its solutions correspond to Newtonian histories of a system of $N$ particles with a total energy $E$ interacting according to the potential $V . T_{\text {kin }}$ looks like the standard Newtonian kinetic energy but note that $\lambda$ represents an arbitrary parameterization of paths in $Q$ : the path length $I$ is invariant under reparameterizations: $\lambda \mapsto \lambda^{\prime}=$ $f(\lambda)$, where $d f / d \lambda>0$ but $f$ is otherwise arbitrary. The equations of motion corresponding to $(2)$ are:

$$
\frac{d}{d \lambda}\left(\sqrt{\frac{F_{E}}{T_{\text {kin }}}} m_{i} \frac{d \vec{x}_{i}}{d \lambda}\right)=\sqrt{\frac{T_{\text {kin }}}{F_{E}}} \frac{\partial F_{E}}{\partial \vec{x}_{i}} .
$$

These simplify dramatically, reducing to the standard form of Newton's second law, if the freedom in the choice of $\lambda$ is exploited to set $F_{E}=T_{\text {kin }}$, i.e., $E=T_{\text {kin }}+V$. The

\footnotetext{
${ }^{76}$ It should be stressed that Barbour initially postulated a Jacobi-like action on purely Machian grounds and only learned of the connections with standard dynamics several years afterwards.
} 
substantivalist sees imposing this requirement as a way to determine the rate at which the system traces out its path in $Q$ relative to a primitive temporal metric. The Machian sees the equation as defining an emergent temporal metric in terms of the temporal parameter that simplifies the dynamics of the system as a whole (Barbour, 1994, 2008).77

Jacobi's principle involves a metric on $Q$. To construct a relational theory, we need a metric on $Q_{\mathrm{RCS}}$. One can be obtained by replacing $T_{\text {kin }}$ (a function of velocities defined with respect to inertial structure) with a function of the relative velocities $\dot{r}_{i j}$. Theories of this kind were independently discovered on a number of occasions during the 2oth century. ${ }^{78}$ They predict mass anisotropy effects (how easy it is to accelerate a body becomes direction dependent) that are ruled out by experiment. It is also not clear how they might generalise to field theory, where analogues of the transtemporal particle identities used in the definition of the $\dot{r}_{i j} \mathrm{~s}$ are absent. Barbour and Bertotti (1982) found a way to surmount both problems.

The (ambitious) relationalist thinks of instantaneous configurations as completely characterised by the ratios of inter-particle separations. A three-dimensional coordinate system encodes such data just if $\left|\vec{x}_{i}-\vec{x}_{j}\right| /\left|\vec{x}_{m}-\vec{x}_{n}\right|=r_{i j} / r_{m n}$ for all particles $i, j, m, n$. If one coordinate system satisfies this constraint, so will any other related to it by a rigid rotation, translation or a dilation (an overall change of scale). The relationalist therefore regards the points of $Q$, not as specifications of positions in some inertial frame, but as natural representations of relational configurations. The representation involves some redundancy: points of $Q$ connected by an element of the similarity group (the group of rigid translations, rotations and dilations) correspond to the same relative configuration. $Q$ is partitioned by the group into sets of such points (the group orbits). Consider, now, two paths in $Q$ that correspond to the same sequence of relative configurations. A metric on $Q$ will, in general, assign them different lengths. However, starting from any given point $p$ in $Q$, one can use the action of the similarity group on $Q$ to define a unique length, by shifting the points of any curve through $p$ along the corresponding group orbits so as to extremize the length assigned to the curve. This is the process Barbour and Bertotti called best matching. ${ }^{79}$ It is depicted in Figure 1 .

\footnotetext{
${ }^{77}$ Note that choosing a simplifying parameter for Equation (4) is quite unlike choosing a time coordinate that is adapted to the spacetime substantivalist's temporal metric. The latter also simplifies the (generally covariant) equations, but these equations explicitly refer to an independent standard of duration. According to the Machian interpretation of Jacobi's Principle, fundamental dynamics is formulated without reference to such an external time. See Pooley (2004, 78-9).

${ }^{78}$ Noteworthy examples are Hofmann (1904), Reissner (1914), Schrödinger (1925), Barbour (1974a), Barbour and Bertotti (1977) and Assis (1989). For further discussion see Earman (1989, 92-6) and Barbour and Pfister (1995 107-78).

${ }^{19}$ For an informal discussion of the central idea, see Barbour (1999. Ch. 7). For the extension to dilations, see Barbour (2003). For more formal and general treatments, see Anderson (2006) and Gryb (2009).
} 


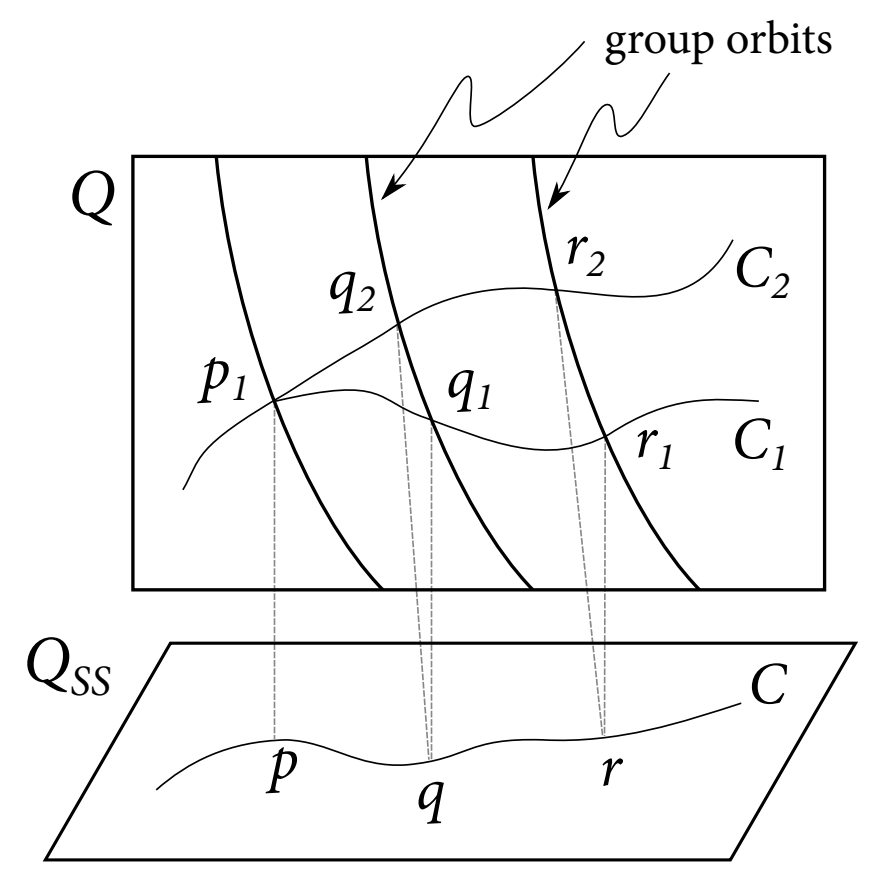

Figure 1: Best Matching. The curves $C_{1}$ and $C_{2}$ in $Q$ correspond to the same sequence of relative configurations. $q_{2}$ is the point on the orbit containing $q_{1}$ that minimises the distance along the curve from $p_{1} . r_{2}$ is similarly related to $q_{2} . C_{2}$ is the bestmatched curve; the length along it gives the length along $C$, the corresponding curve in $Q_{\text {ss. }}$.

If best matching is to define a metric on $Q_{\mathrm{SS}}$ (the quotient of $Q$ by the similarity group), the metric on $Q$ must have the right properties. In particular, suppose $p_{1}$ and $p_{2}$ are points on the same group orbit that are widely separated in $Q$. Consider two paths through $p_{1}$ and $p_{2}$ respectively that correspond to the same sequence of relative configurations. Suppose one now best matches these paths, keeping the points $p_{1}$ and $p_{2}$ fixed. Best matching only leads to a well-defined metric on $Q_{S S}$ if the same result is obtained in each case. The metric $d s^{2}=F_{E} d s_{\text {kin }}^{2}$ satisfies this requirement provided $F_{E}$ meets certain conditions.

If one first considers best matching just with respect to the Euclidean group (translations and rotations), $V$ must be a function only of the relative distances, $r_{i j}$. This requirement is satisfied by familiar Newtonian potentials. The corresponding best-matched theories, which take DPMs to be geodesics of the metric induced on $Q_{\mathrm{RCS}}$, have as solutions sequences of relative configurations that correspond to the standard Newtonian solutions with zero overall angular momentum (relative to the centre-of-mass frame). The fact that a subset of standard Newtonian solutions 
is recoverable by this method highlights the fact that the theories provide a relational interpretation of inertial structure: best matching establishes a non-primitive "equilocality relation", corresponding to the space of the inertial frame in which the system's total linear and angular momenta vanish. Note, also, that the recovery of only a proper subset of the solutions of standard dynamics is arguably a strength of the best-matching theory (assuming solutions capable of modelling the actual world fall within this set). This is because the theory predicts and explains a feature of the world (the vanishing of its overall angular momentum) that is a contingent fact on the orthodox Newtonian view (Pooley and Brown, 2002, Pooley, 2004) ${ }^{80}$

Best matching with respect to dilations imposes a more severe requirement: $F_{E}$ must be a homogeneous function of the $\vec{x}_{i}$ s of degree -2 , in order to compensate for the scaling behaviour of $d s_{\text {kin }}^{2}$. Standard Newtonian potentials do not have this property, but they can nevertheless be incorporated as effective potentials in scale-invariant theories if a weak, epoch-dependent universal force is also included (Barbour, 2003, 1556-7).

Barbour's framework for non-relativistic particle dynamics, therefore, constitutes a genuinely relationalist (and potentially fruitful) alternative to Newtonian physics as standardly conceived. What one is really interested in, though, is how the programme transfers to relativistic physics. The best matching idea can be applied in the context of SR (Barbour and Bertotti, 1982, 302-3), but I move straight to a consideration of GR, where the results are truly surprising. The first step is to consider how one might generalise the framework to configurations manifesting a variably-curved Riemannian geometry. One confronts the issue, raised in Section 6.1.2. of how to deal with the possibility that the geometry of empty space might be both non-trivial and non-reducible to relations between material bodies. Barbour bites the bullet. In the context of GR, the Machian "relationalist" takes the geometry of substantival (instantaneous) space as primitive.

Assume that instantaneous space has the determinate topology of some closed 3-manifold without boundary, $\Sigma$. The obvious analogue of $Q$ is then $\operatorname{Riem}(\Sigma)$, the space of Riemannian 3-metrics on $\Sigma$. An analogue of $Q_{\mathrm{RCS}}$ is superspace: the space of 3-geometries. Two points $\left(\Sigma, h_{a b}\right)$ and $\left(\Sigma, h_{a b}^{\prime}\right)$ of Riem $(\Sigma)$ correspond to the same 3-geometry just if they are isometric, i.e., just if, for some diffeomorphism $d$ of $\Sigma, h_{a b}^{\prime}=d^{*} h_{a b}$. Superspace is therefore $\operatorname{Riem}(\Sigma) / \operatorname{Diff}(\Sigma)$, the quotient of $\operatorname{Riem}(\Sigma)$ by the group of diffeomorphisms of $\Sigma$.

Proceeding as before, one seeks an action principle on superspace defined, via best matching, in terms of a metric on $\operatorname{Riem}(\Sigma)$. In this case, best matching is implemented by diffeomorphisms of $\Sigma$. Seeking as direct a parallel as possible with

\footnotetext{
${ }^{80}$ Similarly, one can argue that the Machian relationalist is able to explain formal features of the potential, such as its dependence only on the $r_{i j} \mathrm{~s}$, that are again nonessential aspects of standard Newtonian theory (Barbour. 2011).
} 
Jacobi's Principle (2) leads to a $\operatorname{Riem}(\Sigma)$ geodesic principle of the form:

$$
\delta I=0, \quad I=\int d \lambda \sqrt{\int d^{3} x \sqrt{h} W \int d^{3} x \sqrt{h} T} .
$$

The first integral inside the square root is the analogue of the conformal factor $F_{E}$ in (2): ${ }^{81}$ the second is the analogue of the (parameterized) kinetic energy. In this case, $T=G_{A}^{a b c d} \dot{h}_{a b} \dot{h}_{c d}$, where $\dot{h}_{a b}=d h_{a b} / d \lambda$ are the metric velocities (with respect to the arbitrary path parameter $\lambda$ ) and the general form of the supermetric $G_{A}^{a b c d}$ is $h^{a c} h^{b d}+A h^{a b} h^{c d}$, where $A$ is an arbitrary constant. Best matching with respect to 3-diffeomorphisms is achieved by replacing $T$ with $T_{\mathrm{BM}}=G_{A}^{a b c d}\left(\dot{h}_{a b}-\mathcal{L}_{\dot{\xi}} h_{a b}\right)\left(\dot{h}_{c d}-\right.$ $\left.\mathcal{L}_{\dot{\xi}} h_{c d}\right)$ and extremizing with respect to variations in $\dot{\xi}^{a}{ }^{82}$

Theories of this kind make good sense but they do not provide direct analogues of GR $\sqrt{83}$ For these, one needs to consider a $\operatorname{Riem}(\Sigma)$ action principle that involves a subtle but radical difference. It has the form:

$$
\delta I=0 \quad I=\int d \lambda \int d^{3} x \sqrt{h W T},
$$

with $W$ and $T$ defined as before. The difference between Principles (5) and (6) is that, in the former, integration over 3-space occurs within a global square root, but in the latter the square root is taken at each point of space and occurs within the spatial integration. ${ }^{84}$ Whereas the reparameterization invariance of $(5)$ gives rise to a single constraint, the position of the square root in (6) leads to an infinity of constraints, one associated with each point of space. These must be propagated by the equations of motion if the theory is to be consistent. This happens only if $A=-1$ in $G_{A}^{a b c d}$ and $W=\Lambda+\alpha R$, where $R$ is the scalar curvature tensor of $h_{a b}$, and $\alpha$ is 0 or \pm 1 . The choice of $\alpha=1$ and the imposition of best matching with respect to 3-diffeomorphisms transforms (6) into the action principle for GR found by Baierlein, Sharp and Wheeler (1962). This is dynamically equivalent to the standard spacetime action restricted to globally hyperbolic spacetimes. In other words, without any spacetime presuppositions and starting with a family of "timeless" action principles for the evolution of 3-geometries of the general form (6), the

\footnotetext{
${ }^{81} W$ can depend on $h_{a b}$ and its spatial derivatives up to some finite order; the presence of $h:=\sqrt{\operatorname{det} h_{a b}}$ is simply to ensure that the integration is invariantly defined.

${ }^{82}$ If $\xi^{a}$ is an infinitesimal 3-vector field, $\left(h_{a b}-\mathcal{L}_{\xi} h_{a b}\right)$ is the result of acting on $h_{a b}$ by an infinitesimal diffeomorphism generated by $\xi^{a} . \mathcal{L}_{\xi} h_{a b}$, the Lie derivative of $h_{a b}$ with respect to $\xi^{a}$, is given by $\mathcal{L}_{\xi} h_{a b}=\nabla_{a} \xi_{b}+\nabla_{b} \xi_{a}$, where $\nabla_{a}$ is the derivative operator associated with the unique torsion-free connection compatible with $h_{a b}$. For the reason why $T_{\mathrm{BM}}$ is defined in terms of the Lie derivatives with respect the velocity of a 3-vector field, see Barbour et al. (2002, 3219) and Barbour (2003, \$4).

$8: \mathrm{Gryb}(2010,16-8)$ contains a brief discussion of these theories.

${ }^{84}$ The importance of the distinction between $(5)$ and $(6)$, and the fact that GR could be cast in the form of (6), was first pointed out to Barbour by Karel Kuchař (Barbour and Bertotti, 1982, 305).
} 
requirement of mathematical consistency alone (almost) uniquely singles out an action principle corresponding to $\mathrm{GR}^{85}$

The BSW action principle for GR, which formally singles out curves in Riem $(\Sigma)$, is degenerate: a point and a direction in $\operatorname{Riem}(\Sigma)$ fail to pick out a unique solution. By itself, this is not a problem for the Machian relationalist. An analogous property holds of the best-matched action principles for particle dynamics: given a point and direction in $Q$, a continuous infinity of curves solve the equations. The reason this is not a drawback in the particle case is that each of these curves corresponds to the same sequence of relative configurations: they project down to a single curve in the quotient of $Q$ by the relevant group. The same is not true for the BSW action. After projecting down from $\operatorname{Riem}(\Sigma)$, one still has a continuum of curves for each point and direction in superspace. Since these curves correspond to non-isometric sequences of 3-geometries, and since such 3-geometries are the Machian's fundamental ontology, the Machian is compelled to regard these curves as corresponding to physically distinct histories. The theory is therefore radically indeterministic. The indeterminism is only removed if one can find a way to regard all curves with the same initial data as representations of a single physical history. As we shall see in Section 7 , the spacetime substantivalist, who regards spatiotemporal geometry as primitive, can do this, because the different sequences of 3-geometries correspond to different foliations of a single 4-dimensional spacetime. The Machian, however, who regards spacetime geometry as secondary to, and defined in terms of, the dynamical evolution of spatial geometry, has no such option (Pooley, 2001. $16-8)$.

Fortunately for the Machian, this otherwise devastating underdetermination can be resolved in strictly 3 -dimensional terms. In the particle case, the ambitious relationalist eschewed transtemporal scale comparisons and regarded only the shapes of configurations as fundamental. Analogous moves are possible in the context of GR. In particular, in a conformal 3-geometry only angles and the ratios of (infinitesimal) distances are regarded as physically fundamental. In terms of Riem $(\Sigma)$, one regards any two 3-metrics related by a (spatially varying) scale transformation as physically equivalent: $h_{a b} \sim \phi h_{a b}, \phi>0$. Conformal superspace is the quotient of $\operatorname{Riem}(\Sigma)$ by such scale transformations (in addition to 3-diffeomorphisms). It can be viewed as analogous to $Q_{s S}$.

Solutions to the BSW action that share initial data in superspace (i.e., sequences

\footnotetext{
${ }^{85}$ This is one of the main results of Barbour et al. (2002), who also claim to recover the equivalence principle and Maxwellian electromagnetism from the constraints that consistency alone places on how matter fields can be added to the theory. The results are extended to Yang Mills theory in Anderson et al. (2003). It should be stressed that these results do not amount to a derivation of GR and the equivalence principle from Machian first principles alone. In addition to the choice of (6) over (5), the form of (6) embodies a number of simplicity assumptions, the relaxing of which permits a range of other Machian theories; see Anderson (2007).
} 
of 3-geometries corresponding to different foliations of the same spacetime) do not project down to a unique curve in conformal superspace. However, the equations of GR can be recast so as to determine a unique such curve (Barbour and Ó Murchadha. 2010). Its points correspond to the foliation of the corresponding spacetime by spacelike hypersurfaces of constant mean extrinsic curvature. The fact that these geometrically privileged hypersurfaces simplify and make tractable the initial value problem in GR has been known since the work of James York in the 1970s. What the Machian perspective provides is an (alternative) understanding of the relevant equations in terms of a generalisation of best matching to (volume preserving) conformal transformations (Anderson et al. 2003, 2005).

The Machian perspective on GR is not relationalist in the sense of this chapter, but it does offer a mathematically and conceptually elegant (and radical) alternative to the standard spacetime perspective. The key issue is not an ontological one about the reality of instantaneous spatial (i.e., spacetime) points (the theory is naturally understood as committed to them); it concerns the relative priority of spatial versus spatiotemporal ideology. Despite Barbour's claims, the local conformal degrees of freedom of CMC spacelike hypersurfaces are not obviously philosophically superior to the standard spacetime quantities: they are not (more) directly observable (recall footnote 36), nor are primitive temporal intervals, or primitive comparisons of distant lengths, somehow inherently suspect. (In fact, an argument could even be made that observability considerations favour spacetime over instantaneous quantities.) Even the parsimony argument in favour of the Machian theory is less clear-cut in GR than in Newtonian mechanics. In GR it is no longer the case that the kinematic structures of the Machian theory are simply a proper subset of those accepted in the spacetime theory ${ }^{86}$ The true test of the Machian programme will be its physical fruitfulness, in particular whether, as its advocates hope, it leads to progress in the search for a theory that successfully reconciles quantum mechanics and general relativity.

\subsection{Have-it-all Relationalism}

The relationalist strategies examined in Sections 6.1 and 6.2 involve a certain honesty. They accept that restricted dynamical symmetries betoken spacetime structure with symmetries that are at least as restricted and seek to square this with relationalism, either by showing how such structure can be both primitive and anchored in a relationalist ontology or by seeking new dynamics with expanded symmetries. The approach reviewed in this section is a case of trying to have one's cake and eat it.

\footnotetext{
${ }^{86}$ There may be good reason to see conformal geometrodynamics as superior to alternative $3+1$ approaches to GR: its basic quantities are dimensionless and the true degrees of freedom are transparent (Barbour 2011, 24, 39). This, though, does not speak directly to the preferability of a $3+1$ over a spacetime perspective.
} 
It seeks a way to reconcile restricted dynamical symmetries with more permissive spacetime symmetries. On this approach, therefore, some of the spacetime structure implicit in the dynamics is judged to have only an effective status, ultimately grounded in a less structured relationalist ontology. Huggett's "regularity approach" is an explicit proposal about how to do this for Newtonian mechanics. The dynamical approach to special relativity, defended by Brown (2005) and Brown and Pooley (2006), can be understood along similar lines.

\subsubsection{The Regularity Approach to Relational Spacetime}

Huggett draws inspiration from some remarks of van Fraassen's on the meaning of Newton's laws. Having posed the problem of how the relationalist can account for the privileged status of the inertial frames, van Fraassen seeks to dissolve it by asserting that inertial frames do not have a privileged status at all (van Fraassen. 1970 116). In claiming this, he is not asserting that Newton's laws fail to differentiate between frames of reference. He acknowledges, of course, that they do. His claim, rather, is that there need be nothing more to the inertial frames' being privileged than their being exactly those frames with respect to which certain statements about mass, motion and force hold true.

The difference between this point of view and the standard substantivalist position might seem elusive, but it concerns which facts are to be taken as basic. For the substantivalist, the basic facts include facts about such things as the relative temporal distances between pairs of events, about what counts as a straight spacetime trajectory, and so on. While the dynamical laws are to be understood in terms of such facts, and while the success of those laws is acknowledged as our only evidence for there being such facts, the facts are not to be conceived of as in any way dependent on the dynamical laws. According to the relationalist view now under consideration, they are so dependent. Beyond the facts that the Leibnizian relationalist acknowledges as primitive, the most basic spatiotemporal fact for van Fraassen is the existence of privileged coordinate systems with respect to which the dynamics of matter takes on a particularly simple form. Non-relational quantities of motion are to be thought of as defined in terms of the very laws in which they feature.

Dynamical laws, and the equations that express them, figure prominently in the characterisation of this position. Exactly what it amounts to, therefore, will depend on how laws themselves are to be conceived. Suppose, for example, that laws of nature are held to involve some kind of primitive natural necessity. The position then becomes the claim that the relative distances between all particles in the universe are constrained as a matter of nomological necessity to evolve over time so that they satisfy certain simple equations with respect to a privileged class of coordinate systems. Such a view, while consistent, has little to recommend it over the substantivalist's acceptance at face value of the quantities featuring in the dynamical 
laws. The relationalist is effectively claiming that relative distances between bodies are constrained to evolve as if each body had an independent quantity of motion that was governed by certain simple laws. This looks like a case where Earman's charge that the relationalist position is "hardly distinguishable from instrumentalism" is justified (Earman, 1989, 128). Whether or not that spells trouble for the relationalist, their debate with the substantivalist has been replaced by a more generic dispute and has lost its distinctive character ${ }^{87}$

Things look more interesting if one adopts a Humean approach to laws. The most promising Humean view is the Mill-Ramsey-Lewis "Best Systems" account, according to which the laws of nature are statements that appear as theorems of those axiom systems true of the totality of Humean facts that best combine the competing virtues of simplicity and strength (see, e.g., Lewis, 1973, 72-3; Earman, 1986, Ch. 5). Without some constraints on admissible vocabulary, the simplicity requirement is not straightforward, because a theory's simplicity appears to be language-dependent. Lewis' later preferred constraint invokes a primitive distinction amongst properties: the formulations of candidate laws with respect to which simplicity is to be judged are in languages whose predicates denote perfectly natural properties and relations (Lewis, 1983). Huggett's idea, effectively, is that this requirement can be liberalised without becoming vacuous. In particular, it is very plausible that, (i) if one assumes the ontology and ideology of Leibnizian relationalism and (ii) if one allows, as candidate Humean laws, systems formulated in terms of supervenient properties as well as perfectly natural properties, Newton's laws will constitute by far and away the simplest and strongest systematisation of a typical Leibnizian relational history compatible with those laws (Huggett, 2006, 48-50).

Unbeknownst to Huggett, a parallel liberalisation of Lewis' Best Systems prescription had already been outlined by Sider, as a possible response to Kripke's 'rotating disks' argument against perdurance (Sider, 2001, 230-4). Sider's goal was to ground a distinction between rotating and non-rotating homogenous matter in the primitive ontology and ideology of the perdurantist (i.e., someone who analyses material persistence in terms of numerically distinct temporal parts of the persisting object located at the different times at which the object exists). The trick is to suppose that Best Systems laws might be formulated in terms of "physical continuants", i.e., aggregates of genidentity-interrelated material events where, crucially, the non-Humean genidentity relation is not a primitive relation but supervenes on the total history of Humean properties together with the laws in which it features:

Consider various ways of grouping stages together into physical contin-

\footnotetext{
${ }^{87}$ One might also worry that if the laws are about coordinate systems it will be hard for the relationalist to avoid what Field (1985) calls heavy duty platonism. (Thanks to Jeremy Goodman for highlighting this.) I take the role of coordinate systems in the specification of the Humean alternative discussed next to be less problematic.
} 
uants. Relative to any such way, there are candidate laws of dynamics. The correct grouping into physical continuants is that grouping that results in the best candidate set of laws of dynamics; the correct laws are the members of this candidate set. (Sider, 2001, 230)

The comparison of Huggett's and Sider's proposals prompts the following worry. If Huggett's reduction of inertial structure relies on primitive transtemporal particle identity and Sider's reduction of material genidentity relies on primitive inertial structure, one or the other of the reductions must be untenable. In response, the liberal Humean might embrace both moves at once: if one strips facts about transtemporal particle identity from a typical Leibnizian relational history compatible with Newton's laws, it remains very plausible that those laws will form part of any Best System theory of such a world, if one is permitted to express the theory in terms of supervenient genidentities with respect to supervenient privileged coordinate systems. But combing both proposals into a single package highlights a related difficulty. Once the strict requirement that primitive vocabulary should express primitive, perfectly natural properties and relations is relaxed, what governs which quantities are part of the supervenience base and which quantities are supervenient? Why stop at a reduction of genidentity and inertial structure? Why not seek to offer a reductive account of mass and charge too? Why not even seek a reductive account of the temporal metric and instantaneous spatial distances? Once the reduction via the dynamical laws of some apparently natural properties to the others is on the table, we need some principles to determine which properties are ripe for reduction and which are to be part of the basic ideology ${ }^{88}$

Huggett himself recognises the issue. He notes that he has included masses and charges but not forces in his supervenience base because "a quantity can only be said to be a force if it plays the right kind of role in the laws and so cannot be metaphysically prior to the laws" (Huggett, 2006, 47). This is a surprising thing for a Humean to say. As Huggett concedes, one might (as many non-Humeans do)

\footnotetext{
${ }^{88}$ If one pursues the programme too far, the supervenience base will eventually become too impoverished to subvene Newtonian laws. Suppose, for example, that the only spatiotemporal information one retains is that which is common to all coordinatizations of the particle trajectories obtainable from an initial inertial coordinate system by smooth but otherwise arbitrary coordinate transformations that preserve the timelike directedness of the trajectories (i.e., that they are nowhere tangent to surfaces of constant time coordinate). Such topological data includes information about whether any two trajectories ever intersect, and information about how the trajectories are 'knotted', but little else. Many Newtonian worlds involving complex histories of relative distances and interactions will be topologically equivalent to histories where all particles maintain constant distance from one another. If one includes only such topological information in the supervenience base, worlds like this will not be worlds where Newton's laws are laws of nature. One might also wonder whether any degree of topological complexity (i.e., any degree of complex entwining of the trajectories) will promote Newton's laws to Best System status. Might not simpler, equally strong alternatives always be available?
} 
say the same about mass and charge. Later, when worrying that his supervenient quantities proposal is "too easy", he cites "serious objections, with a long history, against the supposition of a non-material, physical substance" (ibid., 70) as reason to pursue a reduction that at least allows one to do without spacetime. But, as we have seen, (i) what prima facie strong objections there are to substantivalism can be met and (ii), in the context of Newtonian theory, there are relationalist alternatives to Huggett's programme that do not suffer from this particular problem for regularity relationalism. Huggett does offer a criterion for determining a point beyond which reduction should not be pursued: the laws should be such that they determine the supervenient quantities in all nomically possible worlds (ibid., \$4). However, this does not address the possibility that, with respect to the same set of laws, two distinct sets of putative subvening properties might share this property. In such a case, how does one discover which set contains the "real" fundamental properties?

In his 2006 paper, Huggett only considers Newtonian worlds. We should consider how the programme looks from the perspective of relativistic physics. Special relativity does not provide a very hospitable arena for the view. The relative attractiveness of regularity relationalism in the context of Newtonian physics is due to a couple of factors. First, the fact that the ideology of the Leibnizian relationalist forms a natural subset of Newtonian ideology means that it is relatively natural to seek a reductive account of the additional (inertial) structure in terms of Leibnizian relations. Second, as reviewed in Section 6.1.1, the full neo-Newtonian ideology, when restricted to a (point particle) relationalist ontology, is not sufficient for a relationalist account of standard physics, which underminines one obvious relationalist alternative. Neither factor remains true in the context of SR. In particular, Leibnizian relations are quite unmotivated as a supervenience base; it is far more natural to take the spacetime interval as basic and to understand the spatial distance relations associated with any particular family of simultaneity surfaces in terms of it. Moreover, if the relationalist is happy to countenance spatiotemporal relations between material events as primitive, there is no longer a need for a reduction of some spatiotemporal quantities in terms of others for, as reviewed in Section 6.1.2. the Minkowski interval restricted to material events looks like a viable basis for a relational interpretation of standard specially relativistic physics.

Things are more interesting when one moves to GR. One aspect of Huggett's proposal that I have not so far highlighted is that Huggett sees it as a way to allow the geometry of empty space to supervene on the geometrical relations instantiated by material bodies ${ }^{89}$ Consider, for example, a history of instantaneous spatial relations between bodies that are initially Euclidean but that depart from Euclidicity after some moment, perhaps then to return to Euclidicity after some finite further time. Suppose that this history is a solution of a (generalised) Newtonian theory set in

\footnotetext{
${ }^{89}$ This aspect of the proposal is scrutinised by Belot (2011, Ch. III).
} 
a three-dimensional space, $G$, of a fixed geometry that is everywhere Euclidean except for some finite, geometrically simple, non-Euclidean region. The particles start out in the Euclidean region and eventual stray into the non-Euclidean region. Huggett's idea is that the relationalist can view both the geometry of the total space, and the particles' particular embedding in it, as supervenient on the history of relations via (his liberalised version of) the Best Systems approach to laws. The idea is that the following hypotheses jointly constitute the simplest and strongest systematisation of the relational history: (i) the history of instantaneous relations is constrained to be embeddable at all times into $G$ and (ii) the relational history follows, at any moment, from the instantaneous relations and the embedding into $G$ at that moment, together with a certain set of Newtonian laws. In particular, the simplicity requirement fixes $G$ over other more complicated geometries into which the particular relational history can also be embedded, e.g., geometries with additional non-Euclidean regions unsurveyed by the material particles. ${ }^{90}$

Now recall the problem that the variable geometry of empty regions of spacetime can cause for a relationist who would simply restrict spatiotemporal distance relations to material events: a particular partial history of pseudo-Riemannian relations instantiated within an island configuration of material events, together with the laws of GR, might not fix the future history because of the possible influences of the geometry of empty spacetime beyond the material configuration (Section 6.1.2). In the natural extension of Huggett's scheme, one takes the entire history of instantiated spatiotemporal relations between material events as the supervenience base. One and only one future evolution of the material world compatible with the considered initial segment and laws of GR is, of course, included in this. The interesting question for the liberalising Humean is whether, if facts about the geometry of empty spacetime are allowed to supervene, together with the laws, on the material relational history, the laws of GR will constitute the Best System laws of such a world.

\subsubsection{The Dynamical Approach to Relativity}

I finish this section by highlighting some of similarities between the dynamical approach to special relativity, defended by Brown (2005) and by Brown and Pooley (2006), and Huggett's proposal for Newtonian physics. The dynamical approach seeks to offer a reductive account of the Minkowski spacetime interval in terms of the dynamical symmetries of the laws governing matter. It therefore qualifies as a type of relationalism, although this is not something that Brown himself emphasises.

\footnotetext{
${ }^{90}$ Simplicity will not determine a unique geometry for $G$ in all cases, but Huggett makes a persuasive case that the underdetermination is benign and that the regularity relationalist should be content to live with the possibility that there may be no determinate fact of the matter about the geometry of physical space (Huggett 2006, 55-6).
} 
One of the guiding intuitions behind the dynamical approach concerns explanatory priority. Consider, for example, the relativistic phenomenon of length contraction. Do rods behave as they do in virtue of the spatiotemporal environment in which they are immersed, or are facts about the geometrical structure of spacetime reducible (inter alia) to the behaviour of rods? And if one opts for the latter point of view, what explanation is to be given of why measuring rods in motion are contracted relative to similarly constituted rods at rest?

Brown reads Bell (1976) as seeking to demonstrate that "a moving rod contracts, and a moving clock dilates, because of how it is made up and not because of the nature of its spatio-temporal environment" (Brown, 2005, 8, emphasis in the original). And, Brown thinks, Bell was surely right. This, though, is to present a false dichotomy. The substantivalist should claim that a moving rod's contraction reflects both how it is made up and the nature of its spatiotemporal environment. ${ }^{91}$ Recall the discussion of the explanatory role of substantival geometry in Section 4.3 . The substantivalist should agree that a complex material rod does not conform to the axioms of some geometry simply because that is the substantival geometry in which its is immersed; the rod would not do what it does were the laws governing its microphysical parts different in key respects. But equally, according to the substantivalist, the coordinatedependent equations that are appealed to in, for example, Bell's toy-model derivation of length contraction make implicit reference, via the choice of coordinate system, to primitive spatiotemporal geometry.

What features of the laws governing the constituents of a rod are responsible for the rod's characteristic relativistic behaviour such as its length contraction? In an important sense, the details of the dynamics are irrelevant. If subject to appropriately non-destructive accelerations, rods made of steel, wood and glass will contract by the same amount, and for the same reason, namely, the Lorentz covariance of the laws governing their constituents. ${ }^{92}$ In recent discussion of the

\footnotetext{
${ }^{91}$ This is not to say that every explanatory question one might ask about the phenomenon of length contraction requires an appeal to dynamical laws; in some contexts it is enough to cite the relevant geometrical facts in order to provide an explanation. This is a point explicitly emphasised in Brown and Pooley (2006 78-9, 82), where paradigm explanatory uses of Minkowski diagrams (e.g., to highlight that observers in relative motion consider different cross-sections of a rod's world tube when judging its length) are said to constitute "perfectly acceptable explanations (perhaps the only acceptable explanations) of the explananda in question." Our emphasis of this fact seems to have been overlooked by some authors (Skow 2006. Frisch, 2011).

${ }^{92}$ As it was put in Brown and Pooley (2006, 82): "it is sufficient for these bodies to undergo Lorentz contraction that the laws (whatever they are) that govern the behaviour of their microphysical constituents are Lorentz covariant. It is the fact that the laws are Lorentz covariant... that explains why the bodies Lorentz contract. To appeal to any further details of the laws that govern the cohesion of these bodies would be a mistake." Janssen's (2009) carefully argued case that phenomena recognised to be kinematical (in his sense) should not be explained in terms of the details of their dynamics is therefore one that we had antecedently conceded. The explanation of the phenomena in terms of symmetries nonetheless deserves the label "dynamical" (though not, as acknowledged in Brown and
} 
dynamical approach (e.g., Janssen, 2009:|Frisch, 2011), this point is widely agreed upon. As Frisch emphasises, what genuine disagreement there is centres on the status of the dynamical symmetries to which such explanations appeal.

For Balashov and Janssen, these are ultimately to be explained in terms of the geometry of spacetime. To the question: "Does the Minkowskian nature of spacetime explain why the forces holding a rod together are Lorentz invariant or the other way around?", they reply: "Our intuition is that the geometrical structure of space(-time) is the explanans here and the invariance of the forces the explanandum" (Balashov and Janssen, 2003, 340) and Janssen likes to talk of the symmetries of Minkowski geometry as the common origin of the symmetries of the various laws governing matter. For geometry to play this role, its instantiation in the physical world had better not depend on facts about the dynamical laws. This is true on the substantivalist view reviewed in Section 4.3 but, note, that it is also true on the Minkowski relationalist view discussed in Section 6.1.2, which likewise takes both the ideology of the spacetime interval and its satisfying the constraints of Minkowski geometry as primitive.

How does this alleged explanation of dynamical symmetries in terms of spacetime symmetries go? Clearly it will not be any kind of causal explanation. Moreover, as the examples of Galilean (or Maxwellian) invariant Newtonian physics set in Newtonian (or Galilean) spacetime illustrate $\left.\right|^{93}$ the explanation must be compat ible with the logical possibility of theories in which there is a mismatch between dynamical symmetries and the symmetries of independently postulated spacetime structure (Brown and Pooley, 2006, 83-4).

In these cases, the mismatches are all in one direction; the spacetime symmetries are a proper subset of the dynamical symmetries. It might be thought that the substantivalist can readily explain this. ${ }^{94}$ On their view, dynamical laws ultimately involve coordinate-independent claims describing how dynamically varying matter is constrained by and adapted to spacetime structure. If the properties of spacetime structure are described explicitly, these laws should be expressible by equations that hold good in any coordinate system. But if the spacetime structure has symmetries that allow for a privileged set of adapted coordinate systems, one expects these equations will (apparently) simplify, as some aspects of the spacetime structure will now be encoded in the coordinate system. Recall that, in coordinate terms. dynamical symmetries are transformations between coordinate systems in which

Pooley (2006, 83), "constructive") because the explanantia are (in the first instance) the dynamical symmetries of the laws governing the material systems manifesting the phenomena.

${ }^{93}$ Other examples are provided by Lorentz invariant dynamics set in Newtonian spacetime; see, e.g., Earman (1989, 50-5).

${ }^{94} \mathrm{I}$ am grateful to Hilary Greaves for discussion of this point. The story given here can also be told, mutatis mutandis, by a relationalist who posits primitive spatiotemporal relations held to satisfy primitive geometrical constraints. 
the equations expressing the laws take the same form. If the equations in question are the special, simplified equations, then, on the substantivalist's understanding of these equations, (i) they should hold in all coordinate systems appropriately adapted to spacetime structure, and (ii) they need not hold in others. But, in terms of coordinates, spacetime symmetries just are the transformations between adapted coordinate systems. Hence, the dynamical symmetries should include the spacetime symmetries. And, very crudely, the possibility that dynamical symmetries outstrip spacetime symmetries arises because the dynamical laws governing matter might exploit only some of the spacetime structure, so that the coordinate systems in which dynamics simplifies need be adapted to only some of the structure postulated ${ }^{95}$

Given the substantivalist's understanding of the coordinate-dependent forms of dynamical equations, therefore, it follows that the symmetries of these equations cannot be more restricted than the symmetries of the full set of postulated spacetime structures. In at least this sense, the substantivalist can explain dynamical symmetries in terms of spacetime symmetries. According to the dynamical approach, however, this gets things exactly the wrong way round. Facts about dynamical symmetries come first and are the ground of true claims about the geometry of spacetime: "the Minkowskian metric is no more than a codification of the behaviour of rods and clocks, or equivalently, it is no more than the Kleinian geometry associated with the symmetry group of the quantum physics of the non-gravitational interactions in the theory of matter" (Brown, 2005, 9).

If spacetime geometry is to be grounded in the symmetries of the dynamical laws governing matter, it had better be the case that the very idea of such a law and its symmetries does not presuppose spacetime geometry. That it need not do so is particularly clear if a Humean conception of laws is adopted. This will also bring out the parallels with Huggett's proposal. Recall that Huggett's regularity relationalist postulates primitive Leibnizian relations but no ideology corresponding to inertial structure. The latter is grounded in the existence of a proper subset of the coordinate systems adapted to the Leibnizian relations with respect to which the description of the entire relational history is the solution of particularly simple equations (Newton's laws expressed with respect to inertial frame coordinates). The dynamical approach involves a similar but much more radical move: the metrical relations themselves are to be grounded in exactly the same way.

The idea is best illustrated with a simple example. The advocate of the dynamical approach need not be understood as eschewing all primitive spatiotemporal notions (pace Norton, 2008). In particular, one might take as basic the "topological" extendedness of the material world in four dimensions. Imagine such a world whose only material dynamical entity has pointlike parts whose degrees of freedom can

\footnotetext{
${ }^{95}$ See Earman $(1989,45-7)$ for a related discussion of the connection between spacetime symmetries and dynamical symmetries.
} 
be modelled by the real numbers. One obtains a coordinate description of such an entity by associating, in a way that respects its local topology, each of its pointlike parts with distinct elements of $\mathbb{R}^{4}$, and associating with each of these a real number representing the dynamical state of the corresponding part. In other words, we directly map the parts of the material field postulated to be the sole entity in the world into $\mathbb{R}^{4}$ and choose a way to represent its dynamical state so as to obtain a scalar field on $\mathbb{R}^{4}$. Different choices of coordinate system will yield different mathematical descriptions. Suppose, now, that for some special choice of coordinate system the description one obtains is the solution of a very simple equation. Moreover, suppose that (i) the descriptions one obtains relative to coordinate systems related to this first coordinate system by Lorentz transformations yield (distinct) descriptions that are solutions of the very same equation but that (ii) descriptions with respect to other coordinate systems, if they can be represented as solutions of equations at all, are solutions of more complicated equations.

If all this were the case, the simplest equation might be considered one of the Humean laws of this world ${ }^{96}$ The Lorentz group's being their dynamical symmetry group is constituted by its being the group that maps between the coordinate systems with respect to which descriptions of the material world satisfy the simple equation. And finally, the spatiotemporal geometry of the world is defined in terms of the invariants of the symmetry group so identified. In particular, for the spatiotemporal interval between two parts of the material world $p, q$ to be I just is for $\left(t_{p}-t_{q}\right)^{2}-$ $\left|\vec{x}_{p}-\vec{x}_{q}\right|^{2}= \pm I(p, q)^{2}$ with respect to the privileged coordinate systems. Spacetime geometry is reduced to a notion of dynamical symmetry that does not presuppose it. The example considered is, of course, very simple, and a number of issues will arise when fleshing out an analogous story for more realistic physics. Some of the choices to be made are highlighted by Norton (2008), who denies the feasibility of exactly this kind of project. Two charges he makes are worth dwelling on.

First, he considers the case where the world contains several matter fields, each described by a distinct theory. He grants that each of these might be Lorentz invariant. His challenge to the advocate of the dynamical approach (dubbed the "constructivist") is to justify the assumption that the sets of coordinate systems with respect to which these cases of Lorentz invariance are manifest coincide. The simple answer is that the spatiotemporally coincident parts of distinct matter fields should be assigned the same element of $\mathbb{R}^{4}$. The issue is how this relation of coincidence between matter fields is to be understood. For the substantivalist it involves colocation at the same spacetime point. The Minkowski relationalist, who takes interval facts as primitive, can analyse it in terms of these (though not, of course, straightforwardly

\footnotetext{
${ }^{96}$ This law could be expressed in a coordinate-independent manner if one introduces an auxiliary device, the Minkowski metric, which would then be "no more than a codification of the Kleinian geometry associated with the symmetry group" of the laws.
} 
in terms of the vanishing of the interval, for this will not exclude non-coincident, lightlike related events). What options are open to the constructivist? The most natural is to take spatiotemporal coincidence as primitive (as many relationalists have done; e.g., Rovelli (1997, 194)). After all, the project was to reduce chronogeometric facts to symmetries, not to recover the entire spatiotemporal nature of the world from no spatiotemporal assumptions whatsoever. The constructivist's project might need a primitive notion of "being contiguous", but Norton is wrong to think that it follows from this that constructivists are illicitly committed to the independent existence of spacetime. ${ }^{97}$

The other of Norton's objections that I wish to highlight involves what the constructivist must say about the geometry of empty regions of spacetime and of regions containing homogeneous matter. Suppose some way $K$ of coordinatizing the material world satisfies the type of condition described above. Now suppose that the world contains an empty region of spacetime. Translated into our terms, Norton's point is that any $K^{\prime}$ that agrees with $K$ on its assignment of coordinates to material events will yield the same description. $K^{\prime}$ can differ from $K$ in any way one likes over the coordinates it assigns to the empty region. Does this leave the geometry of the empty region indeterminate? Put this way, that there really is no problem here should be obvious: for the constructivist there is literally nothing in an empty region and so nothing whose geometrical properties might be indeterminate. The constructivist does not believe in the existence of an independently existing spacetime!

The case of homogeneous matter is more problematic. Now one is supposing there are entities-the material pointlike parts of the homogeneous region-whose spatiotemporal relatedness one would like to be able to enquire after. Suppose that the constructivist has attributed some primitive topogical properties to matter. Even so, we can respect these properties and smoothly alter $K$ to $K^{\prime}$ within the region to obtain exactly the same description. The constructivist is forced to conclude that for any two material events in the region there is no fact of the matter concerning the interval between them. How bad is this? Note that a number of other geometrical properties will be determinate (because invariant under all coordinate transformations that leave the description of matter unaltered). For example, the

\footnotetext{
${ }^{97}$ More radical options could also be pursued. Starting with the idea that there are no primitive facts about the contiguity or otherwise of distinct material events, one might nonetheless map them into a single copy of $\mathbb{R}^{n}$. The coincidence of events (which events are to be mapped to the same element in $\mathbb{R}^{n}$ ) is then to be thought of as determined in the same manner as the spacetime interval, i.e., determined by those coordinatizations that yield total descriptions of all events that satisfy some simple set of equations. Perhaps one could even view the value of $n$ (i.e., the dimensionality of spacetime itself) as determined in this way too. As with generalizations of Huggett's proposal (see footnote 88 , the more one views as grounded via some kind of Best System prescription, the more unconstrained the problem becomes; it ceases to be plausible that the complexity of the postulated supervenience base will be sufficient to underwrite the target quantities and the laws they obey.
} 
spacetime volume of the homogeneous region might be determinate even though the spatiotemporal relatedness of the points within it is not ${ }^{98}$ This is surely a peculiarity of the constructivist's position. But, like Huggett's regularity relationalist in the face of analogous problems (Huggett, 2006, 55-6), they might argue that it is not such a painful bullet to have to bite.

\section{Substantivalism in Light of the Hole Argument}

For much of the last 25 years, arguments about spacetime substantivalism have been dominated by discussion of the Hole Argument. This is not the place for a thorough review of the sizeable literature that the argument has spawned. ${ }^{99}$ Here I wish only to highlight one form of substantivalism that evades the Hole Argument and to emphasise an important disanalogy between the Hole Argument and the arguments against Newtonian and Galilean substantivalism that were considered in earlier sections.

Originally due to Einstein, who used it prior to 1915 to explain away his inability (at that point in time) to find satisfactory generally covariant field equations, the Hole Argument was rehabilitated by John Stachel $(1989)$ before being put to work against spacetime substantivalism by Earman and Norton (1987). Let $\mathcal{M}_{1}=\left\langle M, g_{a b}, T_{a b}\right\rangle$ be a model of a generally relativistic theory ${ }^{100}$ It follows from the diffeomorphism invariance of GR that, for an arbitrary diffeomorphism $d, \mathcal{M}_{2}=\left\langle M, d^{*} g_{a b}, d^{*} T_{a b}\right\rangle$ will also satisfy the theory's equations. The natural (though not ineluctable) conclusion is that $\mathcal{M}_{1}$ and $\mathcal{M}_{2}$ jointly represent spacetimes (call them $W_{1}$ and $W_{2}$ ) that are physically possible according to the theory.

In $\mathcal{M}_{1}$, each $p \in M$ is assigned certain properties encoded by $\left\langle g_{a b}(p), T_{a b}(p)\right\rangle$; in $\mathcal{M}_{2}, p$ is assigned the (in general) distinct properties encoded by $\left\langle d^{*} g_{a b}(p), d^{*} T_{a b}(p)\right\rangle$. But, according to the substantivalist, $M$ represent physical spacetime. This means that (on one natural understanding of how the points of $M$ represent physical spacetime points) $\mathcal{M}_{1}$ and $\mathcal{M}_{2}$ represent one and the same spacetime point as having different properties. This gives us the next ingredient in the argument: the claim that the substantivalist is committed to regarding $W_{1}$ and $W_{2}$ as distinct possible worlds ${ }^{101}$

\footnotetext{
${ }^{98}$ Compare how, on some treatments of vagueness, disjunctions can be determinately true (Fred is either bald or not bald) even though neither disjunct is determinately true.

${ }^{99} \mathrm{~A}$ good introduction is provided by Norton (2011).

${ }^{100}$ Recall (section 4 ) that the pseudo-Riemannian metric tensor $g_{a b}$ encodes all of the geometrical properties of spacetime, itself represented by the four-dimensional manifold $M$. Strictly speaking, the stress-energy tensor $T_{a b}$ does not directly represent the fundamental matter content of the model. This will be represented by other fields, in terms of which $T_{a b}$ is defined.

${ }^{101}$ This amounts to the denial of Lebniz Equivalence, Earman and Norton's “acid test" of substantivalism (Earman and Norton, 1987, 521).
} 
The problem is that, if this interpretation of spacetime models is permitted, GR is radically indeterministic. Let $d$ be a hole diffeomorphism, a map that it is only nontrivial within a restricted region of $M$ (the so-called hole). Suppose that, relative to the metric of $\mathcal{M}_{1}, d$ is nontrivial only to the future of some spacelike surface, $\Sigma$. $\mathcal{M}_{1}$ and $\mathcal{M}_{2}$ will then be identical structures up to and including this surface but differ to its future. On the proposed interpretation of $\mathcal{M}_{1}$ and $\mathcal{M}_{2}$, they represent spacetimes that are identical up to the spacelike surface represented by $\Sigma$ but that differ to its future. It follows that the equations of GR, together with a complete specification of the history of the world up to some spacelike surface, fail to fix the future. Earman and Norton do not see this as a problem for substantivalism because they think indeterminism is objectionable per se. Their claim, rather, is that determinism should fail only for reasons of physics and not as the result of a metaphysical commitment and in a theory-independent way (Earman and Norton. 1987, 524).

Note that $\mathcal{M}_{1}$ and $\mathcal{M}_{2}$ are isomorphic structures. The possibilities they represent, therefore, involve exactly the same patterns of qualitative features. If $W_{1}$ and $W_{2}$ are distinct possibilities, they differ only over which spacetime points instantiate which of the particular features common to both worlds. In the terminology of modal metaphysics, the difference between the possibilities is merely haecceitistic (Kaplan 1975). Many of the pro-substantivalist responses to the argument make crucial use of this aspect of the setup.

For example, a substantivalist might agree that accepting GR involves a commitment to such haecceitistic distinctions and accept that the theory is indeterministic. However, they might deny that this indeterminism is in any sense troublesome precisely because it is an indeterminism only about which objects instantiate which properties and not about which patterns of properties are instantiated. A closely related response accepts that GR is committed to haecceitistic distinctions but denies that it follows that GR is indeterministic because the correct definition of determinism, it is claimed, is only sensitive to qualitative differences. ${ }^{102}$

The most popular response, however, has been to advocate some variety of sophisticated substantivalism, i.e., a version of substantivalism that denies the existence of physically possible spacetimes that differ merely haecceitistically. The simplest way to secure this is to endorse antihaecceitism, i.e., the general denial of merely haecceitistic distinctions between possible worlds ${ }^{103}$

\footnotetext{
${ }^{102}$ For further discussion of the definition of determinism appropriate to GR, and of the merits of these options, see Butterfield (1989b), Rynasiewicz (1994), Belot (1995), Leeds (1995), Brighouse (1997) and Melia (1999).

${ }^{105}$ This is my preferred option (see Pooley, 2006, 99-103). Despite the important differences between them, I take Maudlin (1989), Butterfield (1989a), Maidens (1992), Stachel (1993. 2002), Brighouse (1994), Rynasiewicz (1994), Hoefer (1996) and Saunders (2003a) all to deny that the relevant haecceitistic differences correspond to distinct physical possibilities. For several of these
} 
Two arguments discussed earlier in the chapter also involved the claim that, because of the dynamical symmetries of the relevant physical theory, the (relevant stripe of) substantivalist was committed to distinct physically possible worlds, the nonidentity of which was alleged to be problematic. The important difference between these cases and those of the Hole Argument is that the former involve qualitative differences between the relevant worlds. In the case of the kinematic shift, the worlds differ over the absolute velocities assigned to bodies. In the case of Maxwellian invariant dynamics set in Galilean spacetime, they differ over the absolute accelerations assigned to bodies.$^{104}$ The fact that these differences are qualitative has two important consequences.

First, that the possibilities differ qualitatively creates an epistemological problem (given that one cannot observationally distinguish between the relevant quantities) that is not present in the case of merely haecceitistic differences. ${ }^{105}$ Even if diffeomorphic models of GR are to be interpreted as representing distinct possibilities, there is no substantive fact, about which I could be ignorant despite knowing all the observable facts, concerning which model really represents the actual world. Each model is equally apt, and which model represents the actual world will be a matter of (arbitrary) representational convention. In contrast, models of Galilean invariant physics set in Newtonian spacetime that differ by boosts of their material content are not equally suited to represent any given possibility. Even once representational conventions are fixed, the Newtonian substantivalist does not know whether the model that attributes a velocity of $10 \mathrm{~ms}^{-1}$ to the Eiffel Tower, the one that attributes $20 \mathrm{~ms}^{-1}$, or yet some other model, corresponds to the actual world.

Second, the antihaecceitist way out of the Hole dilemma is of no use in the context of the kinematic shift argument. The argument is evaded if any two models related by Galilean boosts can be shown to be different representations of the same state of affairs. Since the models represent qualitatively distinct possibilities according to the Newtonian substantivalist, merely embracing antihaecceitism does not collapse the distinction between them. A substantivalist position that can view Galilean boosted models as distinct representations of one and the same state of

authors (though notably not for Maudlin), the commitment follows from a commitment to some kind of antihaecceitism, at least concerning spacetime points, whether on general philosophical grounds (as in Hoefer's case), or as a perceived lesson of the diffeomorphism invariance of the physics (as in Stachel's case).

${ }^{104}$ Note one parallel between the Hole Argument and the argument against Galilean spacetime that exploits the Maxwell group. The fact that the Maxwell group involves a parameter that is an arbitrary function of time means that the Galilean substantivalist interpretation of the models of a Maxwellian invariant theory involves regarding the theory as indeterministic (cf. Stein, 1977. Saunders, 2003a). The fact that the indeterminism involves qualitative differences (according to the Galilean substantivalist) arguably makes the argument more effective against Galilean substantivalism than the Hole Argument is against GR.

${ }^{105}$ This point is discussed by Horwich (1978), Field (1985) and Maudlin (1993). 
affairs requires substantive work, viz., the replacement of Newton's substantival space with neo-Newtonian spacetime. (A similar observation holds concerning the passage from neo-Newtonian to Newton-Cartan spacetime.) This is in contrast to the so-called static shift argument against Newton's absolute space, which exploits the Euclidean symmetries of Newtonian mechanics and compares only models related by time-independent rotations or translations ${ }^{106}$ In this case antihaecceitism does collapse the number of relevant physical possibilities to one.

It is enough to note that antihaecceitism is a live view within metaphysics in order to see that substantivalism need not fall to the Hole Argument. More controversial is how well-motivated the position is from the perspective of the interpretation of physics. Here a couple of remarks are in order.

First, as Belot and Earman (1999, 2001) have stressed, several physicists grappling with the conceptual and technical problems of unifying quantum mechanics and general relativity do claim to draw substantive morals from the Hole Argument. What is not clear, however, is whether the genuinely substantive interpretational questions that have come to the fore as a result of work on the quantisation of GR have anything to do with the kind of diffeomorphism invariance that lies at the heart of the Hole Argument. One key issue concerns the nature of the "observables" (i.e., the genuine physical magnitudes) of diffeomorphism-invariant theories. Another concerns differences between GR and pre-generally relativistic theories. In particular, are the true physical magnitudes of GR essentially different in kind to those of pre-GR theories (when the latter are properly understood)? Whilst Earman $(2006 a b)$ believes that the right answers to these questions will be inconsistent with anything like a substantivalist interpretation of GR, even of the sophisticated variety, it is not obvious that some of the views about the nature of "observables" advocated by the physicists Earman cites, such as those of Rovelli (2002), are incompatible with sophisticated substantivalism.

Second, some of the work on "structural realist" interpretations of spacetime, at least where these do not involve an eliminativism about spacetime points, can be understood as varieties of antihaecceitist substantivalism ${ }^{107}$ It is possible that the development of one of these will provide a coherent and plausible motivation for

\footnotetext{
${ }^{106}$ An argument like this was made by Leibniz in his correspondence with Clarke (Alexander 1956). That Leibniz makes a precisely parallel argument, exploiting permutation invariance, against the existence of atoms, should give those sympathetic to the static shift argument pause for thought. Consistency should lead one either to embrace or reject both conclusions.

${ }^{107}$ Self-declared structuralist approaches to spacetime that are best described as varieties of substantivalism (in the sense that they include spacetime points amongst the ground-floor ontology) include those of Stachel (2002 2006), Saunders (2003a), Esfeld and Lam (2008) and Muller (2011). For an overview of a wider range of structuralist approaches, see Greaves (2011), who gives reasons to be sceptical that a coherent position that does not collapse into sophisticated substantivalism (or relationalism) has yet to be clearly identified. Bain (2006) is another advocate of spacetime structuralism, not cited by Greaves.
} 
sophisticated substantivalism.

The upshot of this section is that the substantivalist understanding of spacetime physics, as set out in Section 4 , is not undermined by the Hole Argument. What, then, should one conclude about the relative merits of substantivalism versus relationalism? In Section 5 I considered and rejected two other strands of antisubstantivalist argument that have motivated recent relationalists. That leaves substantivalism as a going concern. What about relationalism? Of the three general strategies outlined, the most promising is the Machian, 3-space approach of Barbour and collaborators. But, recall, this turned out not to be a form of relationalism in the traditional, ontological sense. It does represent an approach that is metaphysically very different from spacetime orthodoxy, but the dividing issue is not the existence of spacetime points but the relative priority of 3 -dimensional versus 4 -dimensional concepts.

The other two relationalist approaches fare less well. Recognising that the Maxwell group is a symmetry group of Newtonian physics allows for an intriguing and relatively overlooked form of enriched relationalism, but it does not generalise to relativistic physics. In the context of SR, the restriction of Minkowski distances to a material ontology already provides for a viable, if unexciting, form of relationalism. In the context of GR, however, the same move does not work: in general, the dynamically significant chronometric facts outstrip the chronometric facts about matter, as is most vividly illustrated by the abundance of interesting vacuum solutions.

The relationalist approach reviewed in Section 6.3 has not been pursued in the context of GR. Instead, a popular move for relationalists has been to treat the metric field as just another material field (see, e.g., Rovelli, 1997, 193-5). This, it turns out, is also the view endorsed by Brown (2005. Ch. 9). So, whilst the "dynamical approach" to relativity provides a reductive account of the metric-i.e., is a form of have-it-all relationalism-in the context of SR (Section 6.3.2) the same is not true, for Brown at least, in GR. Brown stresses that the metric field only gains its usual "chronometrical significance" (i.e., only corresponds to the practical geometry

\footnotetext{
${ }^{108} \mathrm{I}$ am attracted to the view that sees individualistic facts as grounded in general facts (Pooley unpublished). However, as Dasgupta (whose terminology I adopt) has recently stressed (Dasgupta 2011. 131-4), this requires that one's understanding of general facts does not presuppose individualistic facts. Since the standard understanding of general facts arguably does take individualistic facts for granted, the spacetime structuralist/sophisticated substantivalist must show that they are not illicitly making the same presupposition. (Dasgupta's own view is that something quite radical is needed (2011 147-52).) The recent literature on "weak discernibility" (see, e.g., Saunders 2003b) has made much of the fact that numerical diversity facts can supervene on facts statable without the identity predicate even when traditional forms of the Principle of the Identity of Indiscernibles are violated. Note, however, that merely showing that one set of facts supervene on another set of facts is not sufficient to show that the former are grounded in the latter (or even that it is possible to think of them as so grounded).
} 
manifest by the behaviour of material rods and clocks) in virtue of the particular way it dynamically couples to matter, but, as I hope to have made clear, no sensible substantivalist should demur.

What, then, is at stake between the metric-reifying relationalist and the traditional substantivalist? Both parties accept the existence of a substantival entity, whose structural properties are characterised mathematically by a pseudoRiemannian metric field and whose connection to the behaviour of material rods and clocks depends on, inter alia, the truth of the strong equivalence principle. It is hard to resist the suspicion that this corner of the debate is becoming merely terminological. At least this much that can be said for the choice of substantivalist language: it underlines an important continuity between the "absolute" spacetime structures that feature in pre-generally relativistic physics and the entity that all sides of the current dispute admit is a fundamental element of reality. To the extent that one should seek to understand the content and success of previous theories in terms of our current best theory, this arguably vindicates the substantivalist interpretation of Newtonian and specially relativistic physics. ${ }^{109}$

\section{References}

Alexander, H. G. (ed.) (1956). The Leibniz-Clarke Correspondence. Manchester University Press, Manchester.

Anandan, Jeeva and Brown, Harvey R. (1995). On the reality of space-time geometry and the wavefunction. Foundations of Physics, 25, 349-60.

Anderson, Edward (2006). Leibniz-Mach foundations for GR and fundamental physics. In General Relativity Research Trends (ed. A. Reimer), Volume 249 of Horizons in World Physics, New York, pp. 59-122. Nova Science. http://arxiv.org/ abs/gr-qc/0405022v2.

Anderson, Edward (2007). On the recovery of geometrodynamics from two different sets of first principles. Studies In History and Philosophy of Modern Physics, 38, $15-57$.

Anderson, Edward, Barbour, Julian B., Foster, Brendan, and Ó Murchadha, Niall (2003). Scale-invariant gravity: Geometrodynamics. Classical and Quantum Gravity, 20, 1571-604.

\footnotetext{
${ }^{109}$ The scepticism concerning the substantiveness of the debate expressed in this paragraph is therefore not that of Rynasiewicz (1996). For a convincing response to many Rynasiewicz's claims, see Hoefer (1998).
} 
Anderson, Edward, Barbour, Julian B., Foster, Brendan Z., Kelleher, B., and Ó Murchadha, Niall (2005). The physical gravitational degrees of freedom. Classical and Quantum Gravity, 22, 1795-802.

Anderson, James L. (1967). Principles of Relativity Physics. Academic Press, New York.

Assis, A. K. T. (1989). On Mach's principle. Foundations of Physics Letters, 2, 301-18.

Baierlein, Ralph F., Sharp, David H., and Wheeler, John A. (1962). Threedimensional geometry as carrier of information about time. Physical Review, 126, 1864-5.

Bain, Jonathan (2004). Theories of Newtonian gravity and empirical indistinguishability. Studies in History and Philosophy of Modern Physics, 35, 345-76.

Bain, Jonathan (2006). Spacetime structuralism. In The Ontology of Spacetime (ed. D. Dieks), Volume 1 of Philosophy and Foundations of Physics, pp. 37-65. Elsevier.

Balashov, Yuri and Janssen, Michel (2003). Presentism and relativity. British Journal for the Philosophy of Science, 54, 327-46.

Barbour, Julian B. (1974a). Relative-distance Machian theories. Nature, 249, 328-9. Misprints corrected in Barbour (1974b).

Barbour, Julian B. (1974b). Nature, 250, 606.

Barbour, Julian B. (1989). Absolute or Relative Motion? Volume 1: The Discovery of Dynamics. Cambridge University Press, Cambridge.

Barbour, Julian B. (1994). The timelessness of quantum gravity: I. the evidence from the classical theory. Classical and Quantum Gravity, 11, 2853-73.

Barbour, Julian B. (1999). The End of Time: The Next Revolution in Our Understanding of the Universe. Weidenfeld \& Nicholson, London.

Barbour, Julian B. (2003). Scale-invariant gravity: Particle dynamics. Classical and Quantum Gravity, 20, 1543-70.

Barbour, Julian B. (2008). The nature of time. http://arxiv.org/abs/0903.3489v1.

Barbour, Julian B. (2010). The definition of Mach's principle. Foundations of Physics, 40, 1263-84.

Barbour, Julian B. (2011). Shape dynamics. an introduction. http://arxiv.org/abs/ 1105.0183v1. 
Barbour, Julian B. and Bertotti, Bruno (1977). Gravity and inertia in a Machian framework. Nuovo Cimento, 38B, 1-27.

Barbour, Julian B. and Bertotti, Bruno (1982). Mach's principle and the structure of dynamical theories. Proceedings of the Royal Society, London, A 382, 295-306.

Barbour, Julian B., Foster, Brendan Z., and Ó Murchadha, Niall (2002). Relativity without relativity. Classical and Quantum Gravity, 19, 3217-48.

Barbour, Julian B. and Ó Murchadha, Niall (2010). Conformal superspace: the configuration space of general relativity. Arxiv preprint: http://arxiv.org/abs/ 1009.3559v1.

Barbour, Julian B. and Pfister, H. (ed.) (1995). Mach's Principle: From Newton's Bucket to Quantum Gravity, Volume 6 of Einstein Studies. Birkhäuser, Boston.

Bell, John S. (1976). How to teach special relativity. Progress in Scientific Culture, $\mathbf{1}$ Reprinted in Bell (1987).

Bell, John S. (1987). Speakable and Unspeakable in Quantum Mechanics. Cambridge University Press, Cambridge.

Belot, Gordon (1995). New work for counterpart theorists: Determinism. British Journal for the Philosophy of Science, 46, 185-95.

Belot, Gordon (1999). Rehabilitating relationism. International Studies in the Philosophy of Science, 13, 35-52.

Belot, Gordon (2011). Geometric Possibility. Oxford University Press, Oxford.

Belot, Gordon and Earman, John (1999). From metaphysics to physics. In From Physics to Philosophy (ed. J. Butterfield and C. Pagonis), pp. 166-86. Cambridge University Press, Cambridge.

Belot, Gordon and Earman, John (2001). Pre-Socratic quantum gravity. In Physics Meets Philosophy at the Planck Scale (ed. C. Callender and N. Huggett), pp. 213-55. Cambridge University Press, Cambridge.

Brighouse, Carolyn (1994). Spacetime and holes. In Proceedings of the $1994 \mathrm{Bi}$ ennial Meeting of the Philosophy of Science Association (ed. D. Hull, M. Forbes, and R. Burian), Volume 1, East Lansing, MI, pp. 117-25. Philosophy of Science Association.

Brighouse, Carolyn (1997). Determinism and modality. British Journal for the Philosophy of Science, 48, 465-81. 
Brown, Harvey R. (2005). Physical Relativity: Space-time Structure from a Dynamical Perspective. Oxford University Press, Oxford.

Brown, Harvey R. and Pooley, Oliver (2006). Minkowski space-time: A glorious non-entity. In The Ontology of Spacetime (ed. D. Dieks), Volume 1 of Philosophy and Foundations of Physics, pp. 67-89. Elsevier, Amsterdam.

Butterfield, Jeremy (1989a). Albert Einstein meets David Lewis. In Proceedings of the 1988 Biennial Meeting of the Philosophy of Science Association, Vol. 2 (ed. A. Fine and J. Leplin), East Lansing, Michigan, pp. 65-81. Philosophy of Science Association.

Butterfield, Jeremy N. (1989b). The hole truth. British Journal for the Philosophy of Science, 40, 1-28.

Catton, Philip and Solomon, Graham (1988). Uniqueness of embeddings and space-time relationalism. Philosophy of Science, 55, 280-91.

Charleton, Walter (1654). Physiologia Epicuro-Gassendo-Charltoniana: Or a Fabrick of Science Natural, Upon the Hypothesis of Atoms, Founded by Epicurus, Repaired by Petrus Gassendus, and Augmented by Walter Charleton. Thomas Newcomb, for Thomas Heath, London.

Dasgupta, Shamik (2011). The bare necessities. Philosophical Perspectives, 25, 115-160.

Descartes, René (1644). Principia Philosophiae. The Philosophical Writings of Descartes, vol. 1, translated and edited by J. Cottingham, R. Stoothoff and D. Murdoch (Cambridge University Press, 1985).

Dieks, Dennis (2006). Another look at general covariance and the equivalence of reference frames. Studies In History and Philosophy of Modern Physics, 37, 174-91.

DiSalle, Robert (1995). Spacetime theory as physical geometry. Erkenntnis, 42, $317-37$.

DiSalle, Robert (2002). Newton's philosophical analysis of space and time. In The Cambridge Companion to Newton (ed. I. B. Cohen and G. E. Smith), pp. 33-56. Cambridge University Press, Cambridge.

DiSalle, Robert (2006). Understanding Space-Time. Cambridge University Press, Cambridge.

Earman, John (1986). A Primer on Determinism. D. Riedel, Dordrecht. 
Earman, John (1989). World Enough and Space-Time: Absolute versus Relational Theories of Space and Time. MIT Press, Cambridge MA.

Earman, John (2006a). The implications of general covariance for the ontology and ideology of spacetime. In The Ontology of Spacetime (ed. D. Dieks), pp. 3-24. Elsevier.

Earman, John (2006b). Two challenges to the requirement of substantive general covariance. Synthese, 148, 443-68.

Earman, John and Norton, John (1987). What price spacetime substantivalism? the hole story. British Journal for the Philosophy of Science, 38, 515-25.

Ehlers, Jürgen (1973a). The nature and structure of space-time. In The Physicist's Conception of Nature (ed. J. Mehra), pp. 71-91. Reidel, Dordrecht, Holland.

Ehlers, Jürgen (1973b). Survey of general relativity theory. In Relativity, astrophysics and cosmology: Proceedings of the summer school held, 14-26 August, 1972 at the Banff Centre, Banff Alberta (ed. W. Israel), Volume 38, pp. 1-125. Kluwer.

Einstein, Albert (1916). The foundation of the general theory of relativity. Annalen der Physik, 49. Reprinted in (Einstein et al., 1952, 109-64).

Einstein, Albert (1918). Prinzipielles zur allgemeinen Relativitätstheorie. Annalen der Physik, 360, 241-4.

Einstein, Albert (1922). The Meaning of Relativity. Princeton University Press, Princeton. Four lectures delivered at Princeton University, May, 1921; translated by E. P. Adams.

Einstein, Albert (1924). Über den Äther. Schweizerische naturforschende Gesellschaft, Verhanflungen, 105, 85-93. Translated by S. W. Saunders in (Saunders and Brown. 1991, 13-20); page references are to this translation.

Einstein, Albert, Lorentz, H. A., Weyl, H., and Minkowski, H. (1952). The Principle of Relativity. Dover.

Esfeld, Michael and Lam, Vincent (2008). Moderate structural realism about spacetime. Synthese, 160, 27-46.

Field, Hartry (1985). Can we dispense with space-time? In Proceedings of the 1984 Biennial Meeting of the Philosophy of Science Association (ed. Asquith and Kitcher), Volume 2, pp. 33-90. 
Friedman, Michael (1983). Foundations of Space-Time Theories: Relativistic Physics and Philosophy of Science. Princeton University Press, Princeton.

Frisch, Mathias (2011). Principle or constructive relativity. Studies in History and Philosophy of Modern Physics, 42, 176-83.

Garber, Daniel (1992). Descartes' Metaphysical Physics. University of Chicago Press, Chicago and London.

Grant, Edward (1981). Much Ado About Nothing: Theories of Space and Vacuum from the Middle Ages to the Scientific Revolution. Cambridge University Press, Cambridge.

Greaves, Hilary (2011). In search of (spacetime) structuralism. Philosophical Perspectives, 25, 189-204.

Gryb, Sean (2009). Implementing Mach's principle using gauge theory. Physical Review D, 80, 024018.

Gryb, Sean (2010). A definition of background independence. Classical and Quantum Gravity, 27, 215018.

Hoefer, Carl (1996). The metaphysics of space-time substantivalism. Journal of Philosophy, 93, 5-27.

Hoefer, Carl (1998). Absolute versus relational spacetime: For better or worse, the debate goes on. British Journal for the Philosophy of Science, 49, 451-67.

Wenzel Hofmann (1995 [1904]). Motion and inertia. In Julian B. Barbour and H. Pfister (eds), Mach's Principle: From Newton's Bucket to Quantum Gravity, volume 6 of Einstein Studies, pages 128-33. Birkhäuser, Boston. Translated by J. B. Barbour from Kritische Beleuchtung der beiden Grundbegriffe der Mechanik: Bewegung und Trägheit und daraus gezogene Folgerungen betreffs der Achsendrehung der Erde des Foucault'schen Pendelversuchs, Vienna and Leipzig: M. Kuppitsch Wwe.

Hood, C. G. (1970). A reformulation of Newtonian dynamics. American Journal of Physics, 38, 438-42.

Horwich, Paul (1978). On the existence of time, space and space-time. Nô̂s, 12, 397-419.

Huggett, Nick (1999). Why manifold substantivalism is probably not a consequence of classical mechanics. International Studies in the Philosophy of Science, 13, 17-34. 
Huggett, Nick (2006). The regularity account of relational spacetime. Mind, 115, $41-73$.

Janssen, Michel (2008). 'No success like failure ...: Einstein's quest for general relativity, 1907-1920. To be published in Janssen and Lehner (forthcoming).

Janssen, Michel (2009). Drawing the line between kinematics and dynamics in special relativity. Studies In History and Philosophy of Modern Physics, 40, 26-52.

Janssen, Michel and Lehner, Christoph (ed.) (forthcoming). The Cambridge Companion to Einstein. Cambridge University Press.

Kaplan, David (1975). How to Russell a Frege-Church. Journal of Philosophy, 72, 716-29.

Koyré, Alexandre (1965). Newtonian Studies. University of Chicago Press, Chicago.

Kretschmann, E. (1917). Über den physikalischen Sinn der Relativitätspostulate. Annalen der Physik, 53, 575-614.

Laymon, Ronald (1978). Newton's bucket experiment. Journal of the History of Philosophy, 16, 399-413.

Leeds, S. (1995). Holes and determinism: Another look. Philosophy of Science, 62, 425-37.

Lehmkuhl, Dennis (2008). Is spacetime a gravitational field? In The Ontology of Spacetime II (ed. D. Dieks), Volume 4 of Philosophy and Foundations of Physics, pp. 83-110. Elsevier.

Lehmkuhl, Dennis (2011). Mass-energy-momentum. Only there because of spacetime? British Journal for the Philosophy of Science, 62, 453-88.

Lewis, David K. (1973). Counterfactuals. Harvard University Press, Cambridge, MA.

Lewis, David K. (1983). New work for a theory of universals. Australasian Journal of Philosophy, 61, 343-77. Reprinted in Lewis (1999).

Lewis, David K. (1999). Papers in Metaphysics and Epistemology. Cambridge University Press, Cambridge.

Lewis, David K. (2000). Causation as influence. The Journal of Philosophy, 97, 182-97.

Mach, Ernst (1919 [1901]). The Science of Mechanics (4th edn). Open Court, LaSalle, Illinois. Translated by T. J. McCormach. 
Maidens, Anna (1992). Review of Earman, John S. [1989]: World enough and spacetime: Absolute versus relational theories of space and time. British Journal for the Philosophy of Science, 43, 129-36.

Malament, David B. (1982). Review of 'Science without numbers: A defense of nominalism' by Hartry H. Field. Journal of Philosophy, 79, 523-34.

Malament, David B. (1995). Is Newtonian cosmology really inconsistent? Philosophy of Science, 62, 489-510.

Malament, David B. (2004). On the time reversal invariance of classical electromagnetic theory. Studies in History and Philosophy of Modern Physics, 35, 295-315.

Malament, David B. (2010). A remark about the "geodesic principle" in general relativity. Available at: http://www. socsci.uci.edu/ dmalamen/bio/papers/GeodesicLaw. pdf.

Malament, David B. (2012). Topics in the Foundations of General Relativity and Newtonian Gravitation Theory. University of Chicago Press. http://www. socsci. uci.edu/ dmalamen/bio/GR.pdf.

Maudlin, Tim (1989). The essence of space-time. In Proceedings of the 1988 Biennial Meeting of the Philosophy of Science Association, Vol. 2 (ed. A. Fine and J. Leplin), East Lansing, Michigan, pp. 82-91. Philosophy of Science Association.

Maudlin, Tim (1993). Buckets of water and waves of space: Why spacetime is probably a substance. Philosophy of Science, 60, 183-203.

Maxwell, James Clerk (1952 [1877]). Matter and Motion. Dover, New York.

Melia, Joseph (1999). Holes, haecceitism and two conceptions of determinism. British Journal for the Philosophy of Science, 50, 639-64.

Melia, Joseph (2003). Modality. Acumen, Chesham.

Mellor, D. H. (1980). On things and causes in spacetime. The British Journal for the Philosophy of Science, 31(3), 282-8.

Minkowski, Hermann (1908). Space and time. pp. 75-96. Dover, New York. Translated by W. Perrett and G. B. Jeffrey.

Misner, Charles W., Thorne, Kip S., and Wheeler, John Archibald (1973). Gravitation. W. H. Freeman and Company, San Francisco.

Muller, Fred A (2011). How to Defeat Wüthrich's Abysmal Embarrassment Argument against Space-Time Structuralism. Philosophy of Science, 78, 1046-1057. 
Mundy, Brent (1983). Relational theories of Euclidean space and Minkowski spacetime. Philosophy of Science, 50, 205-26.

Mundy, Brent (1986). Embedding and uniqueness in relational theories of space. Synthese, 67, 383-90.

Nerlich, Graham (1979). What can geometry explain? The British Journal for the Philosophy of Science, 30, 69-83.

Nerlich, Graham (2010). Why spacetime is not a hidden cause: A realist story. In Space, Time, and Spacetime (ed. V. Petkov), Volume 167 of Fundamental Theories of Physics, pp. 181-91. Springer.

Newton, Isaac (1684 [2004]). De Gravitatione. In Philosophical Writings (ed. A. Janiak), pp. 12-39. Cambridge University Press, Cambridge.

Newton, Isaac (1726 [1999]). Mathematical Principles of Natural Philosophy (third edn). University of California Press, Berkeley. Translated by I. Bernard Cohen and Anne Whitman.

Norton, John D. (1985). What was Einstein's principle of equivalence? Studies In History and Philosophy of Science Part A, 16(3), 203-46.

Norton, John D. (1993). A paradox in Newtonian gravitation theory. In Proceedings of the 1992 Biennial Meeting of the Philosophy of Science Association (ed. M. Forbes, D. Hull, and K. Okruhlik), Volume 2, East Lansing, MI, pp. 412-20. Philosophy of Science Association.

Norton, John D. (2008). Why constructive relativity fails. British Journal for the Philosophy of Science, 59, 821-34.

Norton, John D. (2011). The hole argument. In The Stanford Encyclopedia of Philosophy (Fall $2011 \mathrm{edn}$ ) (ed. E. N. Zalta). http://plato.stanford.edu/archives/ fa112011/entries/spacetime-holearg/.

Patrizi, Francesco (1943). On physical space. Journal of the History of Ideas, 4, 224-245. Translated by Benjamin Brickman.

Pooley, Oliver (2001). Relationism rehabilitated? II: Relativity. http:// philsci-archive.pitt.edu/id/eprint/221

Pooley, Oliver (2004). Comments on Sklar's "relationalist metric of time". Chronos, 6, 77-86. http://philsci-archive.pitt.edu/id/eprint/2915. 
Pooley, Oliver (2006). Points, particles and structural realism. In The Structural Foundations of Quantum Gravity (ed. D. Rickles, S. French, and J. Saatsi), pp. 83-120. Oxford University Press, Oxford. Preprint: http://philsci-archive.pitt. edu/2939/.

Pooley, Oliver (unpublished). Substantivalism and haecceitism. Unpublished manuscript.

Pooley, Oliver (in preparation). The Reality of Spacetime. Oxford University Press, Oxford.

Pooley, Oliver and Brown, Harvey R. (2002). Relationalism rehabilitated? I: Classical mechanics. British Journal for the Philosophy of Science, 53, 183-204. Preprint: http://philsci-archive.pitt.edu/id/eprint/220.

Quine, Willard Van (1951). Ontology and ideology. Philosophical Studies.

Reichenbach, Hans (1924). The theory of motion according to Newton, Leibniz, and Huygens. In Modern Philosophy of Science (ed. M. Reichenbach). Routledge and Kegan Paul (1959).

Reissner, H. (1914). Über die Relativität der Beschleunigungen in der Mechanik [on the relativity of accelerations in mechanics]. Phys. Z., 15, 371-5. Translated by J.B. Barbour in Barbour and Pfister (1995): 134-42.

Rovelli, Carlo (1997). Halfway through the woods: Contemporary research on space and time. In The Cosmos of Science (ed. J. Earman and J. Norton), pp. 180-223. University of Pittsburgh Press, Pittsburgh.

Rovelli, Carlo (2001). Quantum spacetime: What do we know? In Physics Meets Philosophy at the Planck Scale (ed. C. Callender and N. Huggett), pp. 101-22. Cambridge University Press, Cambridge. http://arxiv.org/abs/gr-qc/9903045.

Rovelli, Carlo (2002). Partial observables. Physical Review D, 65, 124013.

Rovelli, Carlo (2004). Quantum Gravity. Cambridge University Press, Cambridge.

Rynasiewicz, Robert A. (1994). The lessons of the hole argument. British Journal for the Philosophy of Science, 45, 407-36.

Rynasiewicz, Robert A. (1995). Absolute vs. relational theories of space and time: A review of John Earman's "World Enough and Space-Time". Philosophy and Phenomenological Research, 55, 675-87. 
Rynasiewicz, Robert A. (1996). Absolute versus relational space-time: An outmoded debate? Journal of Philosophy, 93, 279-306.

Rynasiewicz, Robert A. (2004). Newton's views on space, time, and motion. In The Stanford Encyclopedia of Philosophy (ed. E. N. Zalta).

Saunders, Simon W. (2003a). Indiscernibles, general covariance, and other symmetries. In Revisiting the Foundations of Relativistic Physics. Festschrift in Honour of John Stachel (ed. A. Ashtekar, D. Howard, J. Renn, S. Sarkar, and A. Shimony). Kluwer, Dordrecht. http://philsci-archive.pitt.edu/id/eprint/459.

Saunders, Simon W. (2003b). Physics and Leibniz's principles. In Symmetries in Physics: Philosophical Reflections (ed. K. Brading and E. Castellani), pp. 289-307. Cambridge University Press, Cambridge. http://philsci-archive.pitt.edu/2012/.

Saunders, Simon W. (2012). Rethinking Principia. Unpublished manuscript.

Saunders, Simon W. and Brown, Harvey R. (ed.) (1991). The Philosophy of the Vacuum. Oxford University Press.

Schrödinger, Erwin (1925). Die Erfüllbarkeit der Relativitätsforderung in der klassischen Mechanik [the possibility of fulfilment of the relativity requirement in classical mechanics]. Ann. Phys., 77, 325-36. Translated by J.B. Barbour in Barbour and Pfister (1995, 147-156).

Sider, Ted (2001). Four Dimensionalism: An Ontology of Persistence and Time. Oxford University Press, Oxford and New York.

Sklar, Lawrence (1974). Space, Time and Spacetime. University of California Press, Berkeley.

Skow, Bradford (2006). Physical relativity: Space-time structure from a dynamical perspective. Notre Dame Philosophical Reviews.

Skow, Bradford (2007). Sklar's maneuver. British Journal for the Philosophy of Science, $58,777-86$.

Stachel, John (1989). Einstein's search for general covariance, 1912-1915. In Einstein and the History of General Relativity (ed. D. Howard and J. Stachel). Birkhäuser, Boston.

Stachel, John (1993). The meaning of general covariance. In Philosophical Problems of the Internal and External Worlds: Essays on the Philosophy of Adolph Grünbaum (ed. J. Earman, A. Janis, and G. Massey), pp. 129-6o. University of Pittsburgh Press, Pittsburgh. 
Stachel, John (2002). "the relations between things" versus "the things between relations": The deeper meaning of the hole argument. In Reading Natural Philosophy. Essays in the History and Philosophy of Science and Mathematics (ed. D. B. Malament), pp. 231-66. Open Court, Chicago.

Stachel, John (2006). Structure, individuality and quantum gravity. In Structural Foundations of Quantum Gravity (ed. D. Rickles, S. French, and J. Saatsi), pp. 53-82. Oxford University Press, Oxford.

Stein, Howard (1967). Newtonian space-time. Texas Quarterly, 10, 174-200. Reprinted in Robert Palter, ed., 1970, The Annus Mirabilis of Sir Isaac Newton 1666-1966 (MIT Press, Cambridge, Mass.), pp. 258-284.

Stein, Howard (1977). Some pre-history of general relativity. In Foundations of Space-Time Theories (ed. J. Earman, C. Glymour, and J. Stachel), Volume 8 of Minnesota Studies in the Philosophy of Science. University of Minnesota Press, Minneapolis.

Stein, Howard (1991). On relativity theory and openness of the future. Philosophy of Science, 58, 147-67.

Stein, Howard (2002). Newton's metaphysics. In The Cambridge Companion to Newton, pp. 256-307. Cambridge University Press, Cambridge.

Sus, Adán (2011). On the explanation of inertia.

Synge, John Lighton (1960). Relativity: the general theory. North-Holland Publication Co, Amsterdam.

Teller, Paul (1987). Space-time as a physical quantity. In Kelvin's Baltimore Lectures and Modern Theoretical Physics (ed. P. Achinstein and R. Kagon), pp. 425-448. MIT Press, Cambridge, MA.

Trautman, Andrzej (1962). Conservation laws in general relativity. In Gravitation: An Introduction to Current Research (ed. L. Witten), pp. 169-98. Wiley, New York.

van Fraassen, Bas C. (1970). An Introduction to the Philosophy of Space and Time. Columbia University Press, New York.

Weatherall, James Owen (2011a). The motion of a body in Newtonian theories. Journal of Mathematical Physics, 52.

Weatherall, James Owen (2011b). On the status of the geodesic principle in Newtonian and relativistic physics. http://philsci-archive. pitt.edu/8662/. 
Weinstein, Steven (2001). Absolute quantum mechanics. The British journal for the philosophy of science, 52(1), 67-73.

Westman, Hans and Sonego, Sebastiano (2009). Coordinates, observables and symmetry in relativity. Annals of Physics, 1585-611.

Weyl, Hermann (1922). Space-Time-Matter (4th edn). Methuen and Co. Ltd., London. Translated by H. L. Brose.

Wheeler, John Archibald and Feynman, Richard Phillips (1949). Classical electrodynamics in terms of direct interparticle action. Reviews of Modern Physics, 21, 425-33. 